\title{
Rapid Custom Prototyping of Soft Poroelastic Biosensor for Simultaneous Epicardial Recording and Imaging
}

\section{Bongjoong Kim}

Purdue University

\section{Arvin Soepriatna}

Purdue University

\section{Woohyun Park}

Purdue University West Lafayette https://orcid.org/0000-0002-1799-3104

\section{Abigail Cox}

Purdue University https://orcid.org/0000-0003-2262-8168

\section{Haesoo Moon}

Purdue University West Lafayette

Jianchao Zhao

Los Alamos National Laboratory

\section{Nevin Gupta}

Los Alamos National Laboratory

Chi Hoon Park

Hanyang University

Kyunghun Kim

Purdue University West Lafayette

Hyowon Lee

Purdue University West Lafayette https://orcid.org/0000-0001-7628-1441

\section{Yale Jeon}

Hanyang University

Hanmin Jang

Hanyang University https://orcid.org/0000-0002-7724-4244

\section{Dong Rip Kim}

Hanyang University https://orcid.org/0000-0001-6398-9483

\section{Kwan-Soo Lee}

Los Alamos National Laboratory

\section{Craig Goergen}

Purdue University https://orcid.org/0000-0001-8883-7953

Chi Hwan Lee ( $\sim$ lee2270@purdue.edu ) 


\section{Article}

Keywords: biosensors, geometrical complexities, electrophysiological, DIW

Posted Date: October 15th, 2020

DOl: https://doi.org/10.21203/rs.3.rs-88291/v1

License: (c) (i) This work is licensed under a Creative Commons Attribution 4.0 International License. Read Full License

Version of Record: A version of this preprint was published at Nature Communications on June 17th, 2021. See the published version at https://doi.org/10.1038/s41467-021-23959-3. 


\title{
Rapid Custom Prototyping of Soft Poroelastic Biosensor for Simultaneous Epicardial Recording and Imaging
}

\author{
Bongjoong Kim ${ }^{1 \dagger}$, Arvin H. Soepriatna ${ }^{2 \dagger}$, Woohyun Park', Haesoo Moon ${ }^{2}$, Abigail Cox ${ }^{3}$, \\ Jianchao Zhao ${ }^{4}$, Nevin S. Gupta ${ }^{4}$, Chi Hoon Park ${ }^{4,5}$, Kyunghun Kim ${ }^{2}$, Yale Jeon ${ }^{2,6}$, Hanmin \\ Jang ${ }^{2,6}$, Dong Rip Kim ${ }^{6}$, Hyowon Lee ${ }^{2}$, Kwan-Soo Lee ${ }^{4 *}$, Craig J. Goergen ${ }^{2 *}$, Chi Hwan \\ Lee $^{1,2,7 *}$
}

${ }^{1}$ School of Mechanical Engineering, Purdue University, West Lafayette, IN 47907, USA. ${ }^{2}$ Weldon School of Biomedical Engineering, Purdue University, West Lafayette, IN 47907, USA. ${ }^{3}$ Department of Comparative Pathobiology, Purdue College of Veterinary Medicine, West Lafayette, IN, USA. ${ }^{4}$ Chemical Diagnostics and Engineering, Los Alamos National Laboratory, Los Alamos, New Mexico 87545, USA. ${ }^{5}$ Department of Energy Engineering, Gyeongnam National University of Science and Technology, Jinju-Si 660-758, Republic of Korea. ${ }^{6}$ School of Mechanical Engineering, Hanyang University, Seoul 04763, Republic of Korea. ${ }^{7}$ Department of Materials Engineering, Purdue University, West Lafayette, IN 47907, USA. TThese authors contributed equally to this work. ${ }^{*}$ Correspondence and requests for materials should be addressed to C.H.L. (email: lee2270@purdue.edu) or C.J.G. (email: cgoergen@purdue.edu) or K.-S.L. (email: kslee@lanl.gov).

The growing need for the implementation of stretchable biosensors in the human body and organ systems has driven a new rapid prototyping scheme through the direct ink writing (DIW) of multidimensional functional architectures in an arbitrary shape and size to meet the requirement of adapting the geometric nonlinearity of a specific biological site. Recent approaches involve the use of biocompatible viscoelastic inks that are dispensable through an automated nozzle injection system. However, their pragmatic application remains challenged in particular medical practices that demand long-term reliable recording under periodic large strain cycles, such as the cardiac cycle, due to their viscoelastic nature that produces both mechanical and electrical hysteresis. Herein, we report a new class of a poroelastic silicone composite that is adaptable for highprecision DIW of a custom-designed biosensor, which is exceptionally soft and insensitive to mechanical strain without generating significant hysteresis. The unique structural property of the composite material yields a robust and seamless coupling to living tissues, thereby enabling both high-fidelity recording of spatiotemporal electrophysiological activity and real-time ultrasound imaging for visual feedback. In vivo evaluation of a custom-fit biosensor in a murine acute myocardial infarction model demonstrates a potential clinical utility in the simultaneous intraoperative recording and imaging on the epicardial surface, which may guide a definitive surgical treatment. 
Printed biosensors are integral to the development of various medical research platforms and their broad applications at all scales, from cellular to organ level. ${ }^{1-4}$ Direct ink writing (DIW) of multidimensional functional architectures on various biological substrates enables rapid prototyping and customization of a range of biosensors with geometrical complexities. ${ }^{5-7}$ This approach eliminates the need for multiple steps, masks, and dedicated tools that are typically required in conventional lithography-based techniques. Advanced strategies involve the use of conducting viscoelastic polymers or silicone composites containing conductive nanofillers to serve as dispensable inks for a nozzle injection system that allows for automated rapid prototyping. ${ }^{8-11}$ To this end, it requires precise control of the rheological properties of the inks in order to (1) ensure high-precision printability for sophisticated rendering at the microscale and (2) prevent the hindering of the densely dispersed nanofillers from the polymerization of the inks. ${ }^{12}$ Despite great successes, these materials exhibit mechanical and electrical hysteresis under periodic large strain cycles due to their viscoelastic nature or/and result in irreversible degradation in conductivity due to the difficulty of maintaining the percolation network of the conductive nanofillers. ${ }^{13,14}$ In addition, these materials may produce a risk for delamination from the biological substrates under large deformations due to the low interaction energy at the interface and the discrepancy in their intrinsic elasticity. ${ }^{15}$ The fundamental limitations of these materials impede their implementation in medical practices, particularly under conditions that demand long-term reliable recording against repetitive loading such as the cardiac cycle.

Herein, we introduce a sponge-like form of poroelastic silicone composite with optimal rheological properties that allow it to be printed in a nozzle injection system with high fidelity. The resulting composite material provides the following unique features: (1) poroelastic behavior (rather than viscoelastic behavior) with reversible compressibility that can effectively suppress both mechanical and electrical hysteresis against repetitive loading; ${ }^{16}(2)$ exceptional softness due to the ultralow mechanical modulus $(\mathrm{E}<30 \mathrm{kPa})$ of the sponge-like foam, which is lower than that of commercial dispensable inks (E > 1.11 MPa; SE 1700, Dow Corning) by more than 10-fold and comparable to that of human cardiac tissues $(29-41 \mathrm{kPa}) ;{ }^{17}$ and (3) monolithic structure in which the nanofillers are embedded through the internal pores in a way that eliminates the risk of compromising the structural integrity even under large deformations. ${ }^{18}$ In this report, we elucidated the structure-property relationships of this composite material at the molecular and microsystemic levels and then evaluated its applicability in rapid custom prototyping of stretchable biosensors. To demonstrate the utility of this concept in medical practice, we produced a range of customfit biosensor arrays tailored for simultaneous recording and imaging of acute myocardial infarctions in vivo.

\section{Custom design and prototyping of poroelastic biosensor array}

Figure 1 displays schematic illustrations and the corresponding optical images for the custom design and prototyping of a poroelastic biosensor array. The initial design process began by capturing the overall size, geometry, and structure of the infarcted area of a specific heart through four-dimensional (4D) segmentation (i.e., 3D geometric volume over a cardiac cycle) of the myocardium constructed by lofting sequential short axis (SAX) endocardial and epicardial boundaries from ultrasound images (Figure 1a). ${ }^{19,20}$ Details of the 4D heart segmentation and lofting are shown in the Methods section. This information was taken into consideration to carefully scale and adjust the overall layout of the device in order to meet the requirement of a specific geometric accuracy such that the distance between the pairs of recording electrodes can fit well to the position and orientation of the infarcted area. The subsequent printing process began by directly writing a formulated ink on a Si wafer using an automated nozzle injection system equipped on a three-axis computer-controlled translation stage (Nordson EFD; inner diameter of nozzles $=100 \mu \mathrm{m}$; repeatability $=$ $+/-3 \mu \mathrm{m}$; dispensing rate $=20 \mathrm{~mm} / \mathrm{min}$ ). This setting provides the capability to uniformly render microscale motifs (thickness $\geq 50 \mu \mathrm{m}$-thick; width $\geq 100 \mu \mathrm{m}$-wide) that fit into the pre-designed layout of the device.

Herein, the formulated ink, as described in more detail later, contains an optimal mixture of silicone resins and silica particles to provide both high-precision printability and the capability of turning the ink into a sponge-like foam. As presented in Figure 1b, the selectively patterned traces of the as-printed (prepolymer) ink were polymerized into an amorphous sponge-like morphology with a pore diameter ranging from 5 to $50 \mu \mathrm{m}$ under a pressurized hot steam condition of $120^{\circ} \mathrm{C}$ and 15 psi using a pressure rice cooker (Max, Instant Pot, Inc). Supplementary Figure S1a shows representative results of the differential 
scanning calorimetry (DSC) of the ink to confirm its amorphous character. The formation of the micropores under this condition is likely attributed to the massive penetration and evaporation of the pressurized water molecules to/from the prepolymer. ${ }^{18}$ The resulting sponge-like foam was then immersed in a mixture solution of hexane $(200 \mathrm{ml})$ and $\mathrm{Ag}$ flakes $(0.5 \mathrm{mg}$; $200 \mathrm{~nm}-5 \mu \mathrm{m}$ in diameter; Inframat Advanced Materials, LLC), allowing it to absorb the solution quickly by capillary action in a manner that traps the Ag flakes into the internal pores (Figure 1c). The Ag flakes were then electrolessly plated with $\mathrm{Cu}$ for $\sim 30$ minutes to secure their mechanical and electrical interconnections for which the resulting sheet resistance and stretchability were lower than $7.72 \pm 1.52 \Omega$ and greater than $100 \%$, respectively (Supplementary Figure $\mathrm{S} 1 b)$. The entire surface of the Cu-plated $\mathrm{Ag}$ flakes were also plated with Au for 2 minutes to promote biocompatibility and improve scratch strength. ${ }^{21}$ Next, another direct writing of the formulated ink was followed to define the remaining traces and therefore complete the device in an open mesh layout to ensure breathability and stretchability (Figure 1d). ${ }^{22}$ Finally, a water-soluble medical tape made of polyvinyl alcohol (PVA, Sigma-Aldrich; $50 \mu \mathrm{m}$-thick) was applied to gently peel the device from the Si wafer and then trimmed using scissors to remove excess areas (Figure 1e). The water-soluble medical tape was used as a temporary supporting layer to facilitate its easy integration across the surface of the heart under median sternotomy, which thereafter was dissolved within no more than 30 seconds by applying a warm saline solution. Supplementary Figure S2a presents a series of images for the complete dissolution of the watersoluble medical tape in 10 seconds when inserted into a warm water bath $\left(35^{\circ} \mathrm{C}\right)$. Supplementary Figure $\mathrm{S} 2 \mathrm{~b}$ provides the corresponding results of computational analysis revealing that the total bending stiffness (or flexural rigidity) of the device decreased exponentially as the supporting layer (i.e., water-soluble medical tape) was dissolved over time due to its cubic dependence on thickness. ${ }^{22}$

\section{Material structure-property relationships}

The rheological properties of the formulated ink are key parameters governing the printability and structural integrity of the printed device. ${ }^{12}$ To achieve the optimal rheological properties, we formulated the ink by blending (1) a base resin comprised of vinyl terminated diphenylsiloxane-dimethyl silicone copolymer, methylhydrosiloxane copolymer, and siloxane monomer with the weight ratio of 6.5:3.3:0.2, (2) a dilute resin (Sylgard 184, Dow Corning; 10:1 weight ratio of base and curing agents), and (3) a physical crosslinker of polysiloxane-treated hydrophobic silica $\left(\mathrm{SiO}_{2}\right.$-PS) particles $\left(\mathrm{CAB}-\mathrm{O}-\mathrm{SIL}^{\circledR}\right.$ fumed silica TS-720, CABOT Corp). To predict the miscibility of the blended inks, we first conducted molecular dynamics (MD) simulations and quantitatively evaluated the interfacial interaction energy between the physical cross-linker (i.e., $\mathrm{SiO}_{2}$-PS particles) and the surrounding resins. Figure 2a shows a representative snapshot image of the MD simulation for a $\mathrm{SiO}_{2}$-PS particle, compared to a non-treated (hydroxyl-terminated) silica $\left(\mathrm{SiO}_{2}-\mathrm{OH}\right)$ particle as a control. Details of the MD simulation results are summarized in Table S1. The results show that the interaction energy of the $\mathrm{SiO}_{2}$-PS particle $\left(-1.852 \times 10^{21} \mathrm{kcal} / \mathrm{mol} \cdot \mathrm{g}\right)$ remained substantially lower than the control $\mathrm{SiO}_{2}-\mathrm{OH}$ particle $\left(-6.969 \times 10^{21} \mathrm{kcal} / \mathrm{mol} \cdot \mathrm{g}\right)$, implying that the $\mathrm{SiO}_{2}-\mathrm{PS}$ particle provides enhanced miscibility with the surrounding resins to better serve as a tractable modifier for the rheological properties of the ink. ${ }^{23}$

To characterize the rheological properties, we prepared variously blended inks by varying the weight ratio of the base resin, dilute resin, and $\mathrm{SiO}_{2}$-PS particles from 5.7:3.3:1.0 to 6.0:3.3:0.7, 4.2:5.0:0.8, and 4.5:5.0:0.5, compared to commercial control groups of a dispensable silicone ink (SE 1700, Dow Corning) and a bare PDMS ink (Sylgard 184, Dow Corning). Figure 2b presents the measurement results for the storage (G'; lines) and loss (G”; dotted lines) modulus of these blends. The inks with 5.7:3.3:1.0, 6.0:3.3:0.7, and 4.2:5.0:0.8 ratios and the SE 1700 ink exhibited a gel-like viscoelastic behavior (i.e., G'> G") within the linear viscoelastic (LVE) region (plateau regions) that indicates a printable range without distorting the structural integrity. For example, Supplementary Figure S3 provides a set of images for various as-printed (prepolymer) structures with the minimum linewidth and spacing of $200 \mu \mathrm{m}$ and 300 $\mu \mathrm{m}$, respectively. On the other hand, the ink with 4.5:5.0:0.5 ratio and the Sylgard 184 ink exhibited a liquid-like viscoelastic behavior due to their dominating G" at all shear stresses, which thereby precludes their use for dispensing through a nozzle. The inks exhibited the shear-thinning behavior in which their viscosity decreased with an increase of the shear rate, whereas the Sylgard 184 ink exhibited a zero-shear- 
rate behavior (plateau curve) at all shear rates (Figure 2c). The purple-highlighted area in the graph indicates a range of viscosity where the high-fidelity formation of micropores occurred under the hot pressurized steam condition. The results also show that the ink with 6.0:3.3:0.7 ratio remained within this range at low shear rates. As shown in Figure 2d, this ink also substantially extended the working lifetime (i.e., the time to reach the crossover between G' and G') up to 34 hours, as compared to the SE 1700 ink (2 hours) and the Sylgard 184 ink ( 8 hours). A prolonged working lifetime is essential not only to maintain the structural integrity of the as-printed (prepolymer) ink prior to complete polymerization, ${ }^{24}$ but also to provide sufficient time for the formation of micropores to occur across the entire thickness of the structure up to $150 \mu \mathrm{m}$ (Supplementary Figure S4). For comparison, Supplementary Figure S5 provides the experimental results showing that the formulated ink was completely polymerized after 15 minutes of annealing at $160^{\circ} \mathrm{C}$ (i.e., the plateau curve of G'), whereas the ink mixed with conductive nanofillers (e.g., Ag flakes) remained unpolymerized even after 1 hour of annealing at the same condition. In conclusion, the ink with 6.0:3.3:0.7 ratio meets all requirements of both the appropriate shear-thinning flow behavior and the prolonged working lifetime for the rapid custom prototyping of the sponge-like poroelastic foam.

Figure $2 \mathrm{e}$ shows the stress-strain curve for a printed line $(2 \mathrm{~cm}$-long $\times 2 \mathrm{~mm}$-wide $\times 150 \mu \mathrm{m}$-thick $)$ made of the ink with 6.0:3.3:0.7 ratio before (blue line) and after (red line) the formation of micropores, compared to control groups made of the SE 1700 ink (green line) and the Sylgard 184 ink (black line). The mechanical modulus of the printed line was decreased more than 3-fold $(\mathrm{E}=0.15 \pm 0.02 \mathrm{MPa})$ in the presence of micropores due to their large volumetric porosity $(\sim 70 \%)$, which remained substantially lower than the control groups $(>1.11 \mathrm{MPa})$ by nearly or more than 10 -fold. The inset graph presents the corresponding results obtained from this ink configured into an open mesh layout $(8 \mathrm{~mm} \times 20 \mathrm{~mm})$, providing an ultralow effective mechanical modulus $(29 \pm 12 \mathrm{kPa})$ that is comparable to that of human cardiac tissues $(29-41 \mathrm{kPa}) .{ }^{17}$ These results imply that the printed device provides the capability to gently and intimately interface across the epicardial surface in a way that imposes minimal stress on the tissue. Figure $2 \mathrm{f}$ and $\mathrm{g}$ summarize the mechanical hysteresis and the corresponding energy loss of the printed device under repetitive loading-unloading cycles ( $>5$ times each) at a strain $(\varepsilon)$ of $50 \%$, respectively. The results show that the printed device exhibited substantially suppressed mechanical hysteresis with the lowest energy loss of $4.3 \pm 0.5 \mathrm{~kJ} / \mathrm{m}^{4}$; a value that was substantially lower than the control group made of the SE $1700 \mathrm{ink}\left(23.6 \pm 8.7 \mathrm{~kJ} / \mathrm{m}^{4}\right)$ by more than 5 -fold. This energy loss gap increased proportionally to the strain (Supplementary Figure S6a). Figure $2 \mathrm{~h}$ shows that the electrical hysteresis of the printed device remained substantially low between 0.006 and 0.192 with a strain of up to $30 \%$ that corresponds to the maximum strain of the human heart, ${ }^{25}$ which is also at least 10 -fold lower than similar counterparts reported previously. ${ }^{6,26}$ The printed device was stretched up to nearly $150 \%$ prior to its fracture while maintaining its resistance $\left(R / R_{0}\right)$ below 9.0 (Supplementary Figure $\left.S 6 b\right)$. Figure $2 i$ confirms that the resistance remained constant below 5.0 throughout multiple loading-unloading cycles ( $>1,000$ times) with a strain of up to $30 \%$. Throughout these tests, no evidence of physical separation or the leakage of the conductive nanofillers (i.e., Ag flakes) was observed.

\section{Rapid custom prototyping}

The well-regulated rheological and mechanical properties of the formulated ink drive the exploration for rapid prototyping of custom-designed poroelastic biosensors, in a few hours per batch of a dozen, that can be tailored to cover a wide range of heart sizes and shapes. Figure 3a displays photographs of the customprinted devices, each of which was customized to fit with the enucleated piglet, ovine, porcine, and bovine hearts from upper left to bottom right clockwise. Figure $3 \mathrm{~b}$ schematically illustrates the cross-sectional view of the hearts to compare their sizes. All the devices were able to accommodate the different sizes and shapes of the hearts at various length scales, while simultaneously forming a seamless contact to the irregular surfaces due to their substantially low bending stiffness $\left(<8.0 \times 10^{7} \mathrm{GPa} \cdot \mu \mathrm{m}^{4}\right)$. The poroelastic nature and the open mesh layout of the devices also ensured minimal normal (peeling) stress to the epicardium tissue (i.e., the minimum adhesion energy per unit area $\approx 0.5 \mathrm{~mJ} / \mathrm{m}^{2}$ ) and thereby induced a strong capillary

adhesion at the interface. ${ }^{22}$ These aspects allowed the devices to reliably adhere onto the epicardial surface without slipping and be also detached without significant mechanical impact on the epicardium tissue. 
These observations were consistent when the device was customized and integrated with other organs, such as the enucleated porcine liver (Supplementary Figure S7a). Notably, the quality of the conformal coverage was maintained even when the device was interfaced with the deeply wrinkled surface of a human brain model (Supplementary Figure S7b).

\section{Spatiotemporal recording of epicardial electrocardiogram in vivo}

We evaluated the ability of the custom-printed devices in spatiotemporal recording of epicardial electrocardiogram (ECG) in healthy murine and porcine hearts in vivo. The porcine heart provides a substantial similarity in size and shape to the human heart. ${ }^{27}$ The devices were placed on the left ventricle after median sternotomy using a water-soluble medical tape (i.e., PVA). Following the quick dissolution of the tape with the application of a warm saline solution, the devices ( $50 \mu \mathrm{m}$-thick) adhered intimately to the epicardial surface by capillary adhesion force (Figure 4a). Here, the devices were configured with a total of 4 and 8 pairs of recording electrodes to cover the total areas of $1.25 \mathrm{~cm}^{2}$ and $50 \mathrm{~cm}^{2}$ for the acquisition of murine and porcine ECG signals, respectively. The electrochemical impedance of the individual electrodes $(200 \mu \mathrm{m} \times 200 \mu \mathrm{m})$ was measured in a phosphate-buffered saline solution as $2.1 \mathrm{k} \Omega$ and $1.5 \mathrm{k} \Omega$ at frequencies of $40 \mathrm{~Hz}$ and $150 \mathrm{~Hz}$, respectively (Supplementary Figure S8). The exceptionally soft and thin nature of the devices enabled them to maintain a highly conformal contact to the epicardial surface under normal cardiac cycles with the murine and porcine heart rates of $529.9 \pm 9.3$ and $85.4 \pm 8.5$ beats per minute, respectively. Supplementary Movies S1 shows the representative devices that move synchronously with the underlying epicardium tissue without impeding diastolic function in the murine (left panel) and porcine (right panel) hearts. The degree of conformal coverage across the epicardial surface increased with the decreased thickness of the device from $300 \mu \mathrm{m}$ to $50 \mu \mathrm{m}$ due to the substantially reduced bending stiffness by more than 200-fold (Supplementary Figure S9). Figure 4b shows the corresponding measurement results for the post-processed 3D data of the spatiotemporally recorded murine (left panel) and porcine (right panel) ECG signals. The raw data of the ECG signals is shown in Supplementary Figure S10, displaying a typical ECG tracing of the cardiac cycle that consists of a P-wave (atrial depolarization), a QRS-complex (ventricular depolarization), and a T-wave (ventricular repolarization). The straininsensitive poroelastic behavior (i.e., the negligible electrical hysteresis under cyclic loading with a strain of $<30 \%$ ) of the devices and their robust conformal contact across the beating epicardial surface enabled the high-fidelity acquisition of the ECG signals without noticeable degradation in signal quality over time (Figure 4c, top panel). For comparison, Figure 4c (bottom panel) presents the results of the control measurements obtained simultaneously using a conventional 3-lead electrode set (ERT Control/Gating Module Model 1030, SA Instruments, Stony Brook, NY), clearly displaying the shift (i.e., elevation and depression) of the signal baseline caused by the inhalation and exhalation of breathing, respectively. ${ }^{28}$

\section{Intraoperative epicardial mapping in a murine myocardial infarction model}

Intraoperative epicardial mapping is useful to localize critical regions that indicate the origin of pathophysiological conditions such as arrhythmias after acute myocardial infarction, thereby providing important information to guide definitive surgical treatments, especially when the infarct border needs to be identified. ${ }^{29,30}$ To demonstrate the utility of the custom-printed devices in this surgical setting, we performed intraoperative spatiotemporal mapping of epicardial ECG signals in a murine acute myocardial infarction model in vivo. An adult mouse underwent left thoracotomy to expose the ventral portion of the heart, followed by the placement of a custom-printed (6-channel) sensor array on the epicardial surface to cover the entire ventricular epicardium (Figure 5a). A surgery to permanently ligate the left coronary artery was performed following the same procedures as reported in previous studies. ${ }^{19}$ Representative measurement results of the epicardial ECG signals are shown in Figure 5b. Following approximately 30 seconds of the ligation, acute ST-segment elevation events (red circles in the middle panel) occurred near the ligation point where the sensor channels 2 and 3 were located. After 60 seconds of the ligation, the acute ST-segment elevation events were also detected by the sensor channels 4-6, implying that the regional myocardial infarction propagated toward the apex of the heart with the velocity of approximately 0.6 $\mathrm{mm} / \mathrm{sec}$. The ECG waveforms displayed a convex ST-segment, indicating that the corresponding regions 
experienced ischemia or hypoxic conditions. ${ }^{31}$ Figure $5 \mathrm{c}$ provides the results of the control measurements obtained simultaneously using a 3-lead electrode set, displaying the reciprocal ST-segment depressions that typically appear in conventional monitoring methods using the 3-lead electrode configuration. ${ }^{32}$ The device consistently maintained a robust and intimate coupling to the epicardial surface without changing position throughout the entire recording period ( $>10$ minutes) that involved more than 1,000 individual beats .

The semi-transparency of the device, due to the open mesh layout and thin-film design ( $50 \mu \mathrm{m}$-thick), enabled simultaneous ultrasound mapping, as a means for validating the location and size of the myocardial infarction region in real time. ${ }^{33}$ Figure $5 \mathrm{~d}$ schematically illustrates the experimental setup that includes a high-frequency ultrasound system (Vevo3100, FUJIFILM VisualSonics Inc.), with the enlarged photograph in Supplementary Figure S11. A warmed ultrasound gel (Ultrasound Transmission Gel, Parker, Inc.) was applied directly to the device placed on the left ventricle of the heart. Figure 5e shows a representative short-axis ultrasound image, clearly visualizing the device (yellow circle) along with the epicardium (blue circle) and endocardium (red circle) of the heart. The real-time ultrasound video is shown in Supplementary Movie S2. The results show that the QRS-complex appeared at the initiation of the left ventricle contraction while the P-wave prior to the QRS-complex corresponded to the atrial kick responsible for pushing residual blood from the left atrium to the left ventricle (phase of diastolic left ventricular filling). The overall quality of the ultrasound images and the simultaneously recorded ECG signals was unaffected by motion artifacts (e.g., the heartbeat and respiration) through the robust conformal coupling to the epicardial surface. For comparison, Supplementary Figure S12 provides the ultrasound images of both relatively thick $(200 \mu \mathrm{m}-$ thick; top panel) and thin (50 $\mu$ m-thick; bottom panel) devices placed on the epicardial surface of a fixed murine heart. Supplementary Movie S3 shows the corresponding real-time ultrasound videos during the removal of the devices from the epicardial surface, clearly visualizing the movement of the shadows. These results confirm that the imaging artifacts (e.g., the shadows of the recording electrode pairs) can be minimized with the decreased thickness of the device. Figure $5 \mathrm{f}$ presents the post-processed $3 \mathrm{D}$ image reconstructed from both the ultrasound images and the spatiotemporally recorded ECG signals after 60 seconds of the post-ligation. The continuous real-time display of the 3D images is shown in Supplementary Movie S4, confirming that the acute ST-segment elevation events were detected by the sensor channels 26 (i.e., positive voltages) while the areas nearby the sensor channel 1 remained unaffected (i.e., negative voltages).

\section{Evaluation of biocompatibility, anti-biofouling, and effect on cardiac function}

Evaluation of the in vivo biocompatibility of the custom-printed devices and the effect on cardiac function is a critical factor for demonstrating their long-term engraftment. ${ }^{34}$ To this end, we first evaluated the cellular toxicity and inflammatory response of a printed device. Figure 6a shows the result of a cell compatibility assay for the device that was seeded with heart myoblast (H9C2) cells in a 24-well plate (Fisher Scientific, USA), as measured using a colorimetric assay kit (MTT 3-(4,5-dimethylthiazol-2-yl)2,5-diphenyltetrazolium bromide, Sigma-Aldrich, USA). The results indicate that the proliferation rate of the cells was consistently increased throughout the assay period ( 24 hours), producing no significant difference compared to a control (black bar) and a bare sponge-like foam (green bar). Whereas, the device without the overcoat of $\mathrm{Au}$ (red bar) showed considerably reduced cell variability $(<70 \%)$, suggesting toxicity. Figure $6 \mathrm{~b}$ shows representative histological cross-sectional views of the murine cardiac tissues that were stained with both hematoxylin-eosin (H\&E; left panel) and Masson's trichrome (MTC; right panel) on day 14 post-implant of the device. The results revealed moderate chronic inflammation including the formation of a granuloma for the 14-days implantation. A detailed discussion of the histological analysis is summarized in Supplementary Figure S13 and the Methods section.

Next, we also evaluated the biofouling resistance of the device by quantifying the surface fluorescence intensity after 2 hours of incubation in $6 \mathrm{mg} / \mathrm{ml}$ of a bovine serum albumin-fluorescein conjugate (BSAFITC; A23015, Fisher Scientific, USA) diluted with $1 \times$ phosphate-buffered saline, as compared to control groups made of the SE 1700 ink and the Sylgard 184 ink and prepared on a pristine glass (Figure 6c). Figure $6 \mathrm{~d}$ presents the corresponding results of one-way repeated measures analysis of variance (ANOVA) tests ( $n=5$ per group), showing the statistical differences between groups. The results show that the fluorescence 
intensity of the device $(0.7 \pm 0.5$ a.u.) remained significantly lower than that obtained using the Sylgard 184 ink (10.2 \pm 5.5 a.u.) and the glass $(15.4 \pm 6.1$ a.u. $)$, suggesting that the accumulation of proteins on the surface of the device was effectively prevented due to the microporous structure. ${ }^{35,36}$ The control group made of the SE 1700 ink produced a statistically comparable degree of biofouling resistance (2.8 \pm 1.7 a.u.).

Finally, we evaluated the effect of the device on cardiac function by implanting it on the epicardial surface of the murine heart and then suturing the incision site prior to anesthetic recovery. On days $0,1,7$, and 14 post-implants, we acquired ultrasound images of the left ventricle in a long-axis (LAX) plane (Figure 6e and Supplementary Movie S5). With these LAX ultrasound images, the endocardial surface of the left ventricle was segmented at both end-diastole and peak-systole and used to calculate end-diastolic volume (EDV) and peak-systolic volume (PSV) using the Simpson's rule of discs. ${ }^{37}$ The ejection fraction (i.e., the percentage of blood pumped by the left ventricle during contraction) was then calculated using these volumes to assess global cardiac function. ${ }^{38}$ The results confirm that the ejection fraction of the heart remained within the normal range (60-70\%; green highlighted area) without noticeable decrease throughout the entire implantation period (Figure 6f), which was clearly higher than the abnormal ranges for ischemia reperfusion injury (40-60\%; yellow highlighted area) and permanent ligation (20-40\%; red highlighted area).

\section{Conclusions}

The results reported herein suggest a route towards rapid prototyping of thin and stretchable poroelastic biosensors with a custom-fit design that can meet a specific geometric demand in clinical monitoring. The determination of a new formula for a dispensable silicone ink leads to optimal rheological properties that enable both (1) the high-precision DIW of arbitrary functional microarchitectures at various length scales and (2) the capability of turning the printed microarchitectures into a sponge-like foam in a deterministic manner. The resulting poroelastic biosensor is monolithic in which the densely networked conductive nanofillers are embedded through the internal pores in a way that eliminates the risk of compromising their structural integrity even under large deformations. The poroelastic nature of the biosensors can establish a robust coupling to the epicardial surface and also remain insensitive to periodic cardiac cycle and breathing maneuvers without significant mechanical and electrical hysteresis. The simultaneous intraoperative monitoring of both epicardial ECG and ultrasound signals in a murine acute myocardial infarction model suggests a potential utility of the device for high-fidelity acquisition of real-time 3D cardiac mapping, which may guide surgical interventions such as atrial fibrillation ablation. The in vivo studies also suggest an opportunity to further increase the spatial resolution in order to alleviate the need for reliance on postprocessing algorithms to map the site of acute myocardial infarction in a higher resolution. The basic concept of this approach may be potentially expandable for continuous monitoring of lethal cardiac diseases through chronic implantation of the device and integration with current state-of-the-art means of wirelessly communicating power and data. ${ }^{39}$

\section{References}

1. Loo, J.F.C., Ho, A.H.P., Turner, A.P.F. \& Mak, W.C. Integrated printed microfluidic biosensors. Trends Biotechnol. 37, 1104-1120 (2019).

2. Zhu, Z.J., et al. 3D printed functional and biological materials on moving freeform surfaces. Adv. Mater. 30, 1707495 (2018).

3. Lind, J.U., et al. Instrumented cardiac microphysiological devices via multimaterial threedimensional printing. Nat. Mater. 16, 303-308 (2017).

4. $\mathrm{Xu}, \mathrm{L} . \mathrm{Z}$., et al. Materials and fractal designs for 3D multifunctional integumentary membranes with capabilities in cardiac electrotherapy. Adv. Mater. 27, 1731-1737 (2015).

5. Zhu, Z.J., Park, H.S. \& McAlpine, M.C. 3D printed deformable sensors. Sci. Adv. 6, eaba5575 (2020).

6. Valentine, A.D., et al. Hybrid 3D printing of soft electronics. Adv. Mater. 29, 1703817 (2017).

7. Muth, J.T., et al. Embedded 3D printing of strain sensors within highly stretchable elastomers. $A d v$. Mater. 26, 6307-6312 (2014). 
8. Kraft, U., Molina-Lopez, F., Son, D., Bao, Z.A. \& Murmann, B. Ink development and printing of conducting polymers for intrinsically stretchable interconnects and circuits. Adv. Electron. Mater. 6, 1900681 (2020).

9. Khan, Y., et al. A new frontier of printed electronics: flexible hybrid electronics. Adv. Mater. 32, 1905279 (2020).

10. Molina-Lopez, F., et al. Inkjet-printed stretchable and low voltage synaptic transistor array. Nat. Commun. 10, 2676 (2019).

11. Guo, S.Z., Qiu, K.Y., Meng, F.B., Park, S.H. \& McAlpine, M.C. 3D printed stretchable tactile sensors. Adv. Mater. 29, 1701218 (2017).

12. Daalkhaijav, U., Yirmibesoglu, O.D., Walker, S. \& Menguc, Y. Rheological modification of liquid metal for additive manufacturing of stretchable electronics. Adv. Mater. Technol. 3, 1700351 (2018).

13. Wang, Z.Y., et al. Three dimensional core-shell structured liquid metal/elastomer composite via coaxial direct ink writing for electromagnetic interference shielding. Compos A: Appl Sci Manuf. 136, 105957 (2020).

14. Liu, H., et al. Electrically conductive polymer composites for smart flexible strain sensors: a critical review. J. Mater. Chem. C 6, 12121-12141 (2018).

15. Mei, H.X., Landis, C.M. \& Huang, R. Concomitant wrinkling and buckle-delamination of elastic thin films on compliant substrates. Mech. Mater. 43, 627-642 (2011).

16. Chen, G., et al. Strain- and strain-rate-invariant conductance in a stretchable and compressible 3D conducting polymer foam. Matter 1, 205-218 (2019).

17. Park, J., et al. Electromechanical cardioplasty using a wrapped elasto-conductive epicardial mesh. Sci. Transl. Med. 8, 344ra86 (2016).

18. Jeong, G.S., et al. Solderable and electroplatable flexible electronic circuit on a porous stretchable elastomer. Nat. Commun. 3, 997 (2012).

19. Soepriatna, A.H., et al. Three-dimensional myocardial strain correlates with murine left ventricular remodelling severity post-infarction. J. R. Soc. Interface 16, 20190570 (2019).

20. Updegrove, A., et al. SimVascular: an open source pipeline for cardiovascular simulation. Ann Biomed Eng. 45, 525-541 (2017).

21. Vafaiee, M., Vossoughi, M., Mohammadpour, R. \& Sasanpour, P. Gold-plated electrode with high scratch strength for electrophysiological recordings. Sci. Rep. 9, 2985 (2019).

22. Kim, D.H., et al. Dissolvable films of silk fibroin for ultrathin conformal bio-integrated electronics. Nat. Mater. 9, 511-517 (2010).

23. Lewis, J.A. Direct ink writing of 3D functional materials. Adv. Funct. Mater. 16, 2193-2204 (2006).

24. Ozbolat, V., et al. 3D printing of PDMS improves its mechanical and cell adhesion properties. ACS Biomater. Sci. Eng. 4, 682-693 (2018).

25. Choi, S., et al. Highly conductive, stretchable and biocompatible Ag-Au core-sheath nanowire composite for wearable and implantable bioelectronics. Nat. Nanotechnol. 13, 1048-1056 (2018).

26. Costa, P., Ribeiro, S. \& Lanceros-Mendez, S. Mechanical vs. electrical hysteresis of carbon nanotube/styrene-butadiene-styrene composites and their influence in the electromechanical response. Compos. Sci. Technol. 109, 1-5 (2015).

27. Lelovas, P.P., Kostomitsopoulos, N.G. \& Xanthos, T.T. A comparative anatomic and physiologic overview of the porcine heart. J. Am. Assoc. Lab. Anim. Sci. 53, 432-438 (2014).

28. Sato, M., et al. Simultaneous monitoring of mouse respiratory and cardiac rates through a single precordial electrode. J. Pharmacol. Sci. 137, 177-186 (2018).

29. Cuculich, P.S., et al. The Electrophysiological Cardiac Ventricular Substrate in Patients After Myocardial Infarction. J Am Coll Cardiol 58, 1893-1902 (2011).

30. Liu, J., et al. Intrinsically stretchable electrode array enabled in vivo electrophysiological mapping of atrial fibrillation at cellular resolution. Proc. Natl Acad. Sci. USA. 117, 14769-14778 (2020). 
31. Deshpande, A. \& Birnbaum, Y. ST-segment elevation: Distinguishing ST elevation myocardial infarction from ST elevation secondary to nonischemic etiologies. World J Cardiol 6, 1067-1079 (2014).

32. Arora, N., Mishra, B. \& Ieee. Characterization of a low cost, automated and field deployable 2-lead myocardial infarction detection system. In IEEE International Conference on COMmunication Systems and NETworkS (COMSNETS), 41-46 (2020)

33. $\mathrm{Xu}, \mathrm{L} . \mathrm{Z}$., et al. 3D multifunctional integumentary membranes for spatiotemporal cardiac measurements and stimulation across the entire epicardium. Nat. Commun. 5, 3329 (2014).

34. Bernard, M., Jubeli, E., Pungente, M.D. \& Yagoubi, N. Biocompatibility of polymer-based biomaterials and medical devices - regulations, in vitro screening and risk-management. Biomater. Sci. 6, 2025-2053 (2018).

35. Xu, J.J., Xu, J., Moon, H., Sintim, H.O. \& Lee, H. Zwitterionic porous conjugated polymers as a cersatile platform for antibiofouling implantable bioelectronics. ACS Appl. Polym. Mater. 2, 528536 (2020).

36. Adiga, S.P., et al. Nanoporous materials for biomedical devices. JOM 60, 26-32 (2008).

37. Otterstad, J.E. Measuring left ventricular volume and ejection fraction with the biplane Simpson's method. Heart 88, 559-560 (2002).

38. Lang, R. M. et al. Recommendations for cardiac chamber quantification by echocardiography in adults: an update from the American Society of Echocardiography and the European Association of Cardiovascular Imaging. J. Am. Soc. Echocardiogr 28, 1-39 (2015).

39. Won, S.M., Song, E.M., Reeder, J.T. \& Rogers, J.A. Emerging modalities and implantable technologies for neuromodulation. Cell 181, 115-135 (2020).

\section{Methods}

4D heart segmentation. All ultrasound images were acquired using the Vevo 3100 small animal ultrasound system (FUJIFILM VisualSonics Inc., Toronto, Canada). The 4D ultrasound data (3D geometric volume over a cardiac cycle) of adult mouse hearts with well-developed infarcts were acquired via high frequency ultrasound (FUJIFILM VisualSonics Inc., Toronto, Canada). The cardiac and respiratory gated 2D shortaxis images were acquired from the apex to the base of the left ventricle and spatiotemporally synced to generate the 4D ultrasound data. The reconstructed data was resampled to isotropic voxels and exported to SimVascular for the 3D segmentation at both end-diastolic and peak-systolic timepoints. ${ }^{20}$ STL files of the myocardial wall were then created with uniform meshing and used to design the devices.

Ink composition and preparation. The formulated inks were prepared by blending the following three compositions: a base resin, Sylgard 184, and $\mathrm{SiO}_{2}$-PS silica particle, in a specific weight ratio (5.7:3.3:1.0, 4.2:5.0:0.8, 6.0:3.3:0.7, and 4.5:5.0:0.5) with a mixer (Thinky Mixer) for 5 minutes at 2,000 rpm. The base resin was prepared by blending $64.5 \mathrm{wt} \%$ of vinyl terminated diphenylsiloxane-dimethylsiloxane copolymer (a mixture of 9:1 weight ratio of PDV-0541 and PDV-0525), $33.5 \mathrm{wt} \%$ of trimethylsiloxane terminated methylhydrosiloxane-dimethylsiloxane copolymer (HMS-151), and $2.0 \mathrm{wt} \%$ of 1,3,5,7tetravinyl-1,3,5,7-tetramethylcyclotetrasiloxane (siloxane monomer). Sylgard 184 (a mixture of 10:1 weight ratio of base and curing agent) was used as a dilute resin. The $\mathrm{SiO}_{2}$-PS particles and Pt-carbonyl cyclovinylmethylsiloxane complex (Pt catalyst; $0.1 \mathrm{wt} \%$ of the amount of base resin) were added and blended using a mixer (Thinky Mixer) for 2 minutes at 2,000 rpm to adjust the rheological properties of the ink. The control inks (e.g., Sylgard 184 and SE 1700) were prepared by mixing the base and curing agent at the standard 10:1 weight ratio.

Mechanical and electrical measurements under cyclic strains. The well-mixed blends for the formulated inks with a certain ratio were spin-casted on a water-soluble film (PVA, Sigma-Aldrich, $10 \mu \mathrm{m}$-thick) to form a thin layer of the thickness of $150 \mu \mathrm{m}$. Any air bubbles introduced during the casting process were removed before placing the lids on the molds. Following polymerization at $120^{\circ} \mathrm{C}$ for 30 minutes in an oven, the specimens were allowed to cool down to room temperature for about 15 minutes and then trimmed 
into a rectangular shape with the width of $2 \mathrm{~mm}$. Tensile strength was measured using a mechanical testing system (Mark-10). The specimens were loaded into uniaxial grips and then pulled to reach a break point at a speed of $20 \mathrm{~mm} / \mathrm{min}$. A total of 5 trials per specimen was taken, and the corresponding standard deviation was reported as a measurement error. For the electrical measurement, a source meter (Keithley 2400, Tektronix) and a custom-built LabView code (National Instruments) were used in a two-wire configuration.

Measurement of rheological properties. Rheological measurements were carried out on a Rheometer (Discovery Hybrid Rheometer-2, TA Instrument) using parallel plate fixtures with a diameter of $25 \mathrm{~mm}$. The viscosity and shear moduli were measured by stress sweeps ranging from 0.1 to $65,000 \mathrm{~Pa}$ at a fixed angular frequency of $10 \mathrm{rad} / \mathrm{sec}$ for each measurement. Oscillatory time sweeps were measured to investigate gel-points (i.e., working lifetime), where the storage modulus (G') becomes larger than the loss modulus (G”) at an angular frequency of $10 \mathrm{rad} / \mathrm{sec}$ and a strain rate of $4 \%$.

Molecular dynamics (MD) simulation. The Forcite and Amorphous Cell modules in Material Studio (BIOVIA, UK) were used for the MD simulation. The density of the surface treated-layer, composed of one vinyl terminated diphenylsiloxane-dimethylsiloxane copolymer and three trimethylsiloxane terminated methylhydrosiloxane-dimethylsiloxane copolymer main-chains, was set to $0.97 \mathrm{~g} \cdot \mathrm{cm}^{-3}$. Geometryoptimized 3D models were followed by the anneal protocol in which the temperature of the system was sequentially set to $298,398,498$, and $598 \mathrm{~K}$, and then decreased in reverse using a constant-volume ensemble (NVT). Each step was performed for $50 \mathrm{ps,} \mathrm{and} \mathrm{the} \mathrm{anneal} \mathrm{protocol} \mathrm{was} \mathrm{repeated} 5$ times. The interaction energies of the 3D models were obtained after additional NVT dynamics at $298 \mathrm{~K}$ for 1,000 ps. The Condensed-phase Optimized Molecular Potentials for Atomistic Simulation Studies (COMPASS) II force field, the Ewald summation method for non-bonding interactions with an accuracy of $0.001 \mathrm{kcal} \mathrm{mol}^{-}$ ${ }^{1}$, the time step of $1.0 \mathrm{fs}$, the Andersen temperature control method with 1 as the collision ratio, and the Berendsen pressure control method with $0.1 \mathrm{ps}$ as the decay constant were chosen.

Fabrication of custom-printed sensor array. The process began with a $\mathrm{Si}$ wafer coated with a thin layer $(1 \mu \mathrm{m}$-thick) of polymethyl methacrylate (PMMA) as a chemically-dissolvable sacrificial layer. The surface of the PMMA layer was exposed with 3-Aminopropyltriethoxysilane (APTES) in a vacuum desiccator to form a hydrophilic silane group to improve the adhesion strength. A direct writing of the formulated ink was followed using the nozzle injection system (Nordson EFD). For the formation of micropores, the asprinted (prepolymer) ink was annealed in a pressure rice cooker (Max, Instant Pot, Inc.) in which the steam temperature and pressure were set at $120^{\circ} \mathrm{C}$ and $15 \mathrm{psi}$ with the ramping rate of $15^{\circ} \mathrm{C} / \mathrm{min}$ and $5.6 \mathrm{psi} / \mathrm{min}$, respectively. Next, the resulting structure was immersed in a mixture solution $(200 \mathrm{ml})$ of hexane and $\mathrm{Ag}$ flakes (200 nm-5 $\mu \mathrm{m}$ in diameter; Inframat Advanced Materials, LLC) to trap the Ag flakes into the internal pores. The structure was then immersed in a $\mathrm{Cu}$ plating solution that contains (1) copper(II) sulfate pentahydrate $\left(\mathrm{CuSO}_{4} \cdot 5 \mathrm{H}_{2} \mathrm{O}\right.$, Sigma-Aldrich; $\left.18 \mathrm{~g} / \mathrm{L}\right)$, (2) Ethylenediaminetetraacetic acid (EDTA, SigmaAldrich; $48 \mathrm{~g} / \mathrm{L})$, (3) Potassium hexacyanoferrate(II) trihydrate $\left(\mathrm{K}_{4}\left[\mathrm{Fe}(\mathrm{CN})_{6}\right] \cdot 3 \mathrm{H}_{2} \mathrm{O}\right.$, Sigma-Aldrich; 600 $\mathrm{mg} / \mathrm{L})$, (4) Sodium Hydroxide $(\mathrm{NaOH}$, Fisher scientific; 45g/L), (5) Poly(ethylene glycol) $\left(\mathrm{H}\left(\mathrm{OCH}_{2} \mathrm{CH}_{2}\right)_{\mathrm{n}} \mathrm{OH}\right.$, Sigma-Aldrich; 500mg/1L), (6) Formaldehyde (HCHO, Fisher scientific; $\left.20 \mathrm{~mL} / \mathrm{L}\right)$, and $(7)$ hydrochloric acid solution $(1 \mathrm{~N})(\mathrm{HCl}$, Fisher Scientific; $18 \mathrm{~mL} / \mathrm{L})$ for 30 minutes. The Cu-plated surface was subsequently plated with $\mathrm{Au}$ for 2 minutes using an electroplating system (24K Pure gold plating solution-Bath, Gold Plating Services), followed by thorough rinsing with deionized (DI) water. A layer ( $\sim 50 \mu \mathrm{m}$-thick) of polyvinyl alcohol (PVA, Sigma-Aldrich) was drop-casted to form the temporary handling support. Following the complete dissolution of the bottom PMMA layer with acetone, the complete device was physically separated from the Si wafer by gently peeling the PVA layer. Trimming the excess area of the PVA layer completed the entire process.

Animal surgeries on mice. All surgical procedures on mice were performed aseptically and approved by the Purdue Animal Care and Use Committee under protocol number 1505001246. Adult male mice ( $>12$ weeks old; wild-type; C57BL/6J; The Jackson Laboratory, Bar Harbor, ME) were used for this study. To 
prepare for surgery, each mouse was anesthetized with 1-3\% isoflurane delivered in $100 \% \mathrm{O}_{2}$ and endotracheally intubated to a small animal ventilator (SomnoSuite, Kent Scientific, Torrington, CT). To prevent pneumothorax, a pressure-controlled ventilation was employed to maintain a target inspiratory pressure of $18 \mathrm{~cm} \mathrm{H}_{2} \mathrm{O}$ and a peak-end expiratory pressure of $5 \mathrm{~cm} \mathrm{H}_{2} \mathrm{O}$ in the lungs. The mouse was secured to a heated surgical stage, and the body temperature was kept between $36^{\circ} \mathrm{C}$ and $37^{\circ} \mathrm{C}$ using a homeothermic control module. A 3-lead needle electrode set (ERT Control/Gating Module Model 1030, SA Instruments, Stony Brook, NY) was positioned in a Lead I configuration to continuously collect ECG waveforms throughout the surgical procedure at a sampling rate of $900 \mathrm{~Hz}$. A small incision was made along the third intercostal space of the left thorax, and the ribs were retracted to expose the left ventricle before dissecting the pericardium to identify the left coronary artery. For acute infarction studies $(n=5)$, an 8-0 nylon suture was loosely looped around the left coronary artery, and the testbed custom-printed devices were placed on the epicardial surface using a water-soluble medical tape (PVA; $50 \mu \mathrm{m}$-thick, Sigma-Aldrich, USA). Warmed sterile saline was applied to completely dissolve the water-soluble tape within no more than 30 seconds. A suture was tightened to permanently ligate the left coronary artery in order to induce an acute infarct. Successful ligation was confirmed by discoloration of the myocardium in regions distal to the ligation site. ECG data from both the printed devices and the control 3-lead electrodes were acquired simultaneously and synchronized by their timestamps. The mice in the acute infarction group were euthanized humanely at the end of the procedure. For implantation surgeries $(n=3)$, we placed the printed device $(50 \mu \mathrm{m}$-thick) to the epicardial surface as mentioned previously, sutured close the incision site, and allowed the mice to recover. Buprenorphine $(0.05 \mathrm{mg} / \mathrm{kg}$ animal body weight $)$ was administered via intraperitoneal injection as an analgesic. The left ventricles of these mice were imaged with ultrasound on days 1, 7, and 14 post-implant, and one mouse was euthanized at each time point for longitudinal histological data.

Animal surgeries on pigs. All surgical procedures on pigs were terminal, performed by a trained veterinary team from Purdue University's College of Veterinary Medicine, and approved by the Purdue Animal Care and Use Committee under protocol number 1406001099. Adult domestic pigs $(n=2)$ were anesthetized and intubated with a ventilator throughout the entire procedure. A median sternotomy was conducted and the pericardium was cut to visualize the anterior wall of the left ventricle. The custom-printed devices, configured with a total of 8 pairs of recording electrodes, were then placed onto the epicardial surface of the left ventricle using a water-soluble medical tape. Warmed sterile saline was applied to dissolve the water-soluble tape for a tight conformal contact, and ECG waveforms were recorded continuously.

Simultaneous ultrasound imaging during ECG recording. All ultrasound images were acquired using the Vevo 3100 small animal ultrasound system (FUJIFILM VisualSonics Inc., Toronto, Canada). For the preparation prior to imaging, mice were anesthetized with 1-3\% isoflurane delivered in medical-grade air and positioned supine on a heated imaging stage, with paws secured to gold-plated stage electrodes to monitor for ECG and respiration signals. A $40 \mathrm{MHz}$ central frequency linear array transducer with 256 elements (22-55MHz; MX550D) was then positioned on the left ventral thorax to acquire ultrasound images in multiple long and short-axis planes. For the data acquisition, both ECG and respiratory gatings were implemented to minimize breathing artifacts and to acquire cardiac cine loops at 1,000 Hz. To evaluate left ventricular function, the endocardial surface of the left ventricle was manually segmented at end-diastole and peak-systole to approximate left ventricular volumes using the Simpson's rule of discs. ${ }^{38}$ Ejection fraction $(\mathrm{EF})$ was then calculated as follows:

$$
\mathrm{EF}=\frac{\mathrm{EDV}-\mathrm{PSV}}{\mathrm{EDV}} \times 100
$$

where EDV and PSV correspond to end-diastolic volume and peak-systolic volume, respectively. For the in vivo open chest imaging, a warmed ultrasound gel was applied directly onto the epicardially-implanted device on the beating heart at the end of several infarction surgeries. Several representative short-axis 
images of the left ventricle were acquired to visualize adherence of the patch to the left ventricle. For the ex vivo ultrasound imaging on fixed tissues, the devices (50-300 $\mu \mathrm{m}$-thick) were placed on the epicardial surface of the previously fixed left ventricles. An ultrasound gel was used as a conductive medium between the transducer and the fixed tissue. Multiple short-axis images of the left ventricles were acquired to investigate how the devices impact on the quality of ultrasound imaging.

Anti-biofouling analysis. The biofouling resistance of the printed devices was analyzed following the same procedures as reported in a previous study. ${ }^{35}$ The specimens were incubated in bovine serum, fluorescein conjugate (BSA-FITC; $6 \mathrm{mg} / \mathrm{ml}$ ) diluted in $1 \times$ phosphate-buffered solution for 2 hours in a 6 -well plate protected from light at room temperature ( $\mathrm{n}=5$ per group). The specimens were then rinsed with $1 \times$ phosphate-buffered solution prior to air-drying and imaging. The fluorescence z-stack images were captured at 10× magnification using an inverted fluorescence microscope (Axio Observer Z1, Carl Zeiss Microscopy, Jena, Germany). The fluorescence intensity was quantified using a Java-based image processing program (ImageJ). All images were acquired using the same exposure settings to ensure no overor under-saturated pixels (pixel values $=0-255$ ) or image histogram aberrations. The mean intensity and standard deviation were measured from whole image selections, and the statistical analysis was performed with the SAS software (SAS Institute).

Histological analysis. At the end of the implantation experiment, mice were euthanized humanely. A retrograde perfusion was performed from the inferior vena cava using $30 \mathrm{mM}$ potassium chloride $(\mathrm{KCl})$ solution to clear residual blood and arrest the heart in diastole. The heart was harvested while minimizing any disruption to the implanted device on the epicardial surface and then fixed in $4 \%$ paraformaldehyde for 7 days at $4^{\circ} \mathrm{C}$ before sending the samples for histology. The hearts were embedded in paraffin, thinly sectioned (5 $\mu \mathrm{m}$-thick), and stained with hematoxylin-eosin (H\&E) and Masson's trichrome (MTC). The stained tissues were imaged in segments at 10× magnification using a Leica ICC50W stereomicroscope (Leica Microsystems Inc., Buffalo Grove, IL), and stitched together with MosaicJ. Microscopic examination was performed by a board-certified veterinary pathologist and the interpretation was based on standard histopathological morphology of a mouse heart. Four serial transverse sections of the heart from the base to the mid-papillary region were histologically evaluated. The results revealed that, on day 7 postimplant, the implanted device was present adjacent to the wall of the aorta invoking the formation of a granuloma (Supplementary Figure 13a). The granuloma was comprised of a central necrotic core of eosinophilic cellular and karyorrhectic debris mixed with numerous degenerate and viable neutrophils. Surrounding the necrotic core were epithelioid macrophages and multinucleated giant cells, rimmed peripherally by lymphocytes, plasma cells, fibroblasts, and fibrous connective tissue. The epicardium of the right and left atrium was expanded by fibroblasts admixed by neutrophils, lymphocytes and macrophages (Supplementary Figure 13b, left panel). Fragments of the foreign material surrounded by inflammatory cells were found adjacent to the wall of the right atrium. On day 14 post-implant, the epicardium of the right ventricle was thickened with pale eosinophilic collagen fibers and increased fibroblast cellularity. The pericardium was similarly thickened with collagen, numerous fibroblasts, small caliber blood vessels and infiltrates of lymphocytes, plasma cells, macrophages, multinucleate giant cells and few neutrophils. The thickened pericardium surrounded fragments of granular, black foreign material with multifocal adhesions to the underlying epicardium (Supplementary Figure 13b, right panel). The observed lesions predominantly bordered the right ventricular free wall. The implant elicited a chronic state by day 14 mostly centered towards the right side of the heart (Supplementary Figure 13c). The pericardium was only observed on the right-side following tissue processing, likely because the pericardium was adhered to the underlying inflamed epicardium. In summary, chronic inflammation due to a foreign body response was present after long-term implantation. While short-term intraoperative implantation side effects cannot be ruled out, we observed no issues during intraoperative epicardial mapping. 


\section{Data availability}

The data that support the plots and other findings of this study are available from the corresponding authors upon reasonable request.

\section{Acknowledgments}

We thank Bill Schoenlein and Melissa Bible for their help with the porcine surgeries. C.H.L. acknowledges funding support from the Asian Office of Aerospace Research \& Development (AOARD: FA2386-16-14105; program manager: Dr. Tony Kim) and the Air Force Office of Scientific Research (AFOSR: FA238618-1-40171; program manager: Dr. Tony Kim). K.-S.L. acknowledges support from the US Department of Energy's National Nuclear Security Administration contract DE-AC52-06NA25396 and the Dynamic Materials Properties Campaign. The Los Alamos National Laboratory is an affirmative action equal opportunity employer, managed by Triad National Security, LLC for the U.S. Department of Energy's NNSA, under contract 89233218CNA000001. H.L. acknowledges funding support from the National Science Foundation (United States) under grants ECCS-1944480 and CNS-1726865. C.H.P. acknowledges funding support from the National Research Foundation of Korea (NRF) Grant funded by the Korea government (MSIT) (NRF-2017R1C1B3009270 and 2019R1A2C1087209). D.R.K. acknowledges funding support from the International Research and Development Program (NRF-2018K1A3A1A32055469) and the Basic Science Research Program (NRF-2018R1C1B6007938) through the National Research Foundation of Korea (NRF) funded by the Ministry of Science and ICT of Korea.

\section{Author contributions}

C.H.L., C.J.G., and K.S.L. conceived the concept, planned the project, and supervised the research. C.H.L., C.J.G., K.S.L., B.K, A.H.S., and W.P. designed and conducted the experiments and data analysis. J.Z., N.S.G., K.K., Y.J., H.J., and D.R.K. characterized the mechanical and electrical properties of the devices. W.P., A.C., H.M., H.L., and A.H.S. evaluated the biocompatibility, biofouling, and effect on cardiac function of the devices. C.H.P. developed the numerical codes and carried out the computational simulations. C.H.L., C.J.G., K.S.L., B.K., and A.H.S. wrote the manuscript. All authors analyzed the data and commented on the paper.

\section{Supplementary Movie Captions}

Movie S1. Real-time video of the device placed on the epicardial surface of a murine (left panel) and porcine (right panel) heart.

Movie S2. Real-time ultrasound video of the device placed on the epicardial surface of a murine heart.

Movie S3. Real-time ultrasound videos during the removal of the relatively thick ( $200 \mu \mathrm{m}$-thick; top panel) and thin $(50 \mu \mathrm{m}$-thick; bottom panel) devices from the epicardial surface of a fixed murine heart.

Movie S4. Real-time display of the post-processed 3D images reconstructed from the spatiotemporally recorded ECG and ultrasound signals for $0.3 \mathrm{sec}$ after the ligation.

Movie S5. Real-time ultrasound videos of the murine heart on days 1, 7, and 14 post-implant of the device. 


\section{Table of Contents}

High-precision direct ink writing of custom-fit microporous biosensors is demonstrated for robust and seamless coupling to the epicardial surface in vivo. Real-time spatiotemporal mapping of both electrocardiography and ultrasound signals is enabled through the unique poroelastic nature of the biosensors, providing exceptional softness, strain-insensitive conductance, optical semitransparency, and anti-biofouling properties.

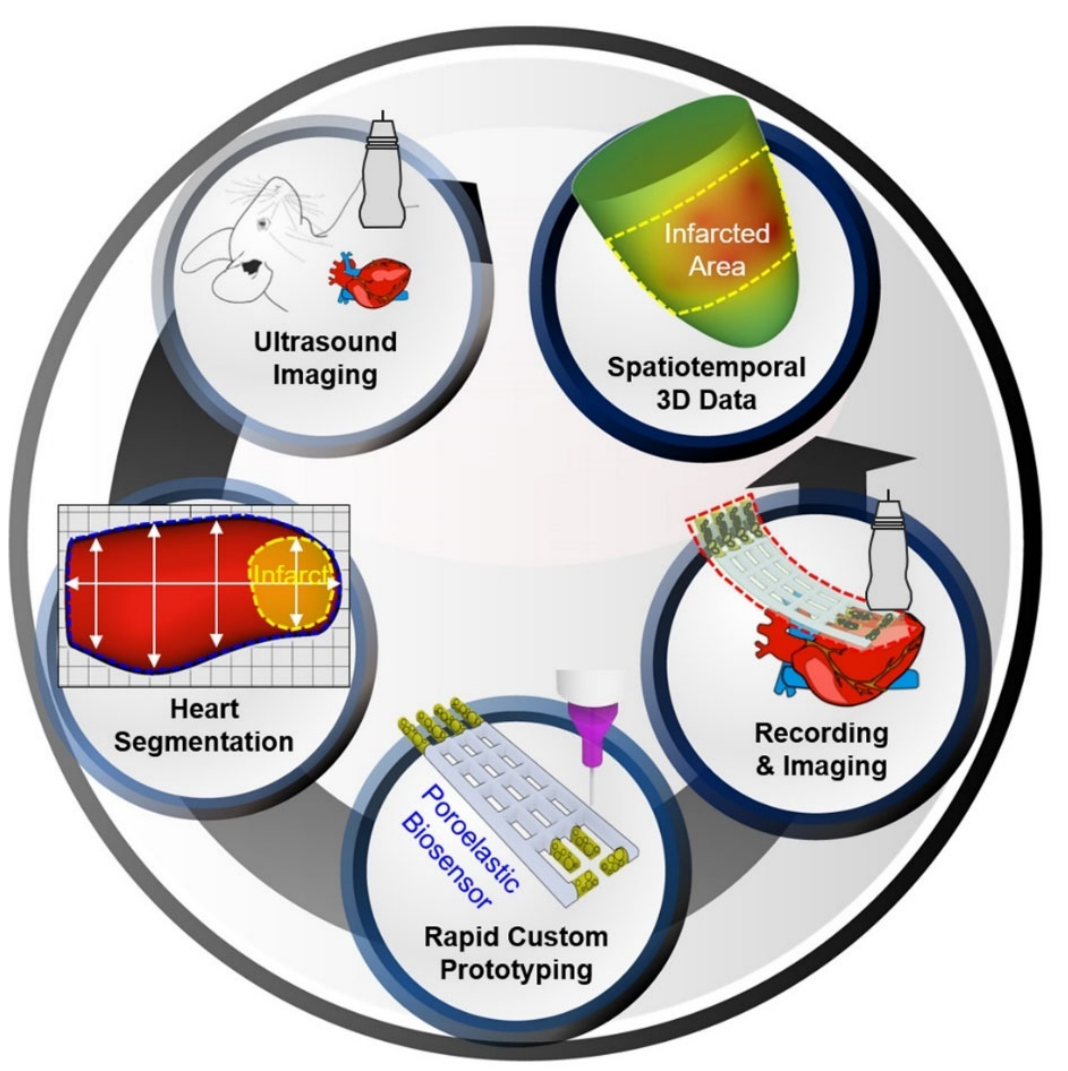




\section{Heart Segmentation \&
a Custom Direct Ink Writing (DIW)}
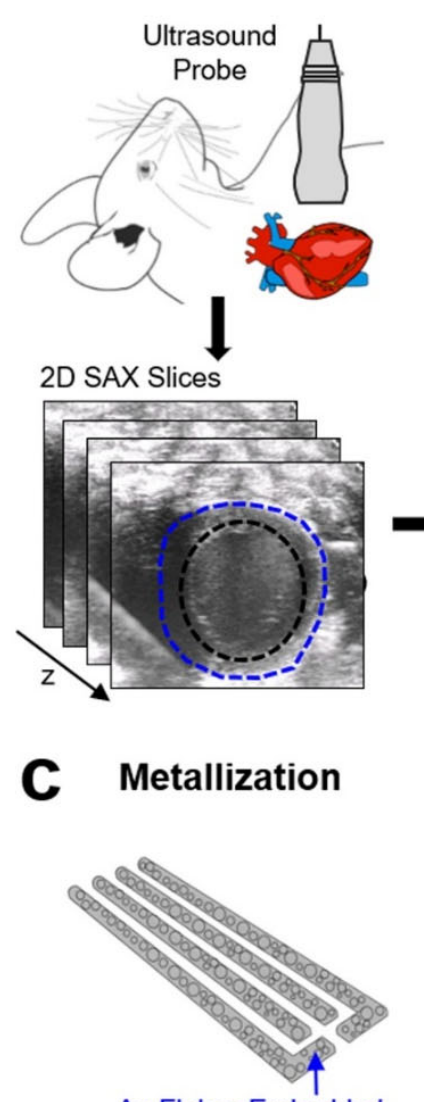

Ag Flakes Embedded in Micropores

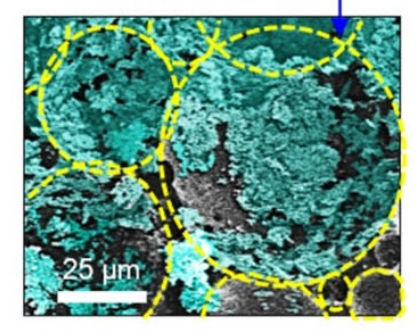

Post-DIW
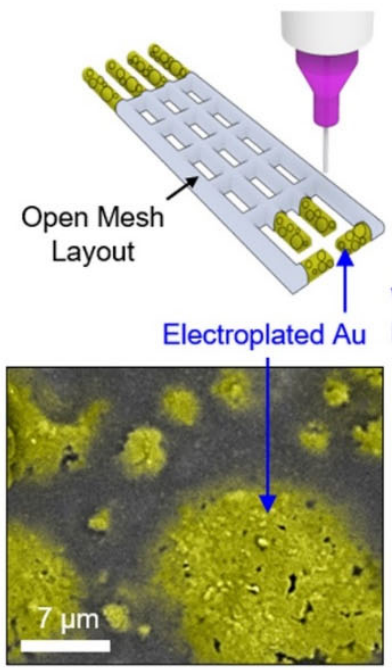

b Steam Etching

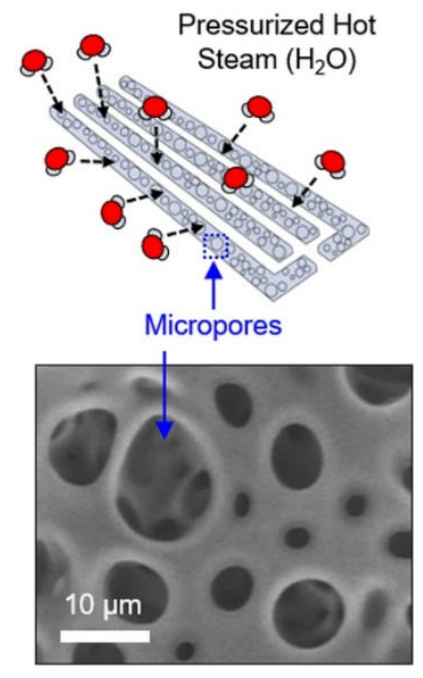

e Implementation

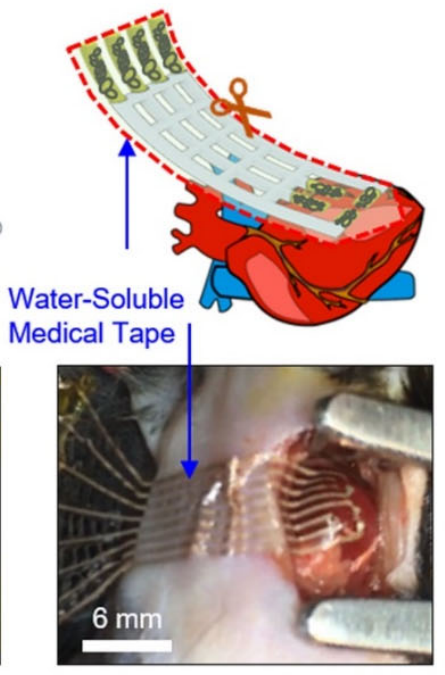

Figure 1. Custom design and prototyping of poroelastic biosensor array. Schematic illustrations and optical images for the key process steps: (a) 3D imaging and custom direct ink writing (DIW), (b) steam etching, (c) metallization, (d) post-DIW, and (e) implementation on the epicardial surface of a murine heart. 
a

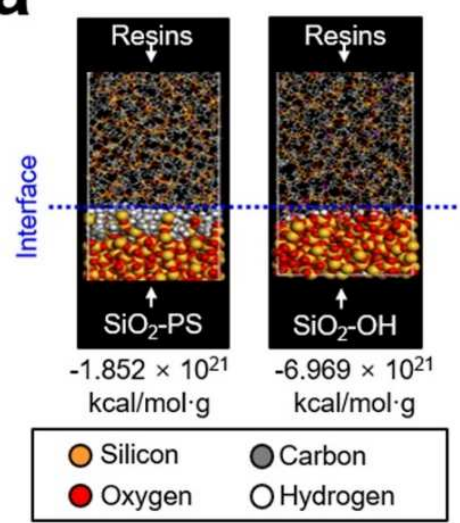

d
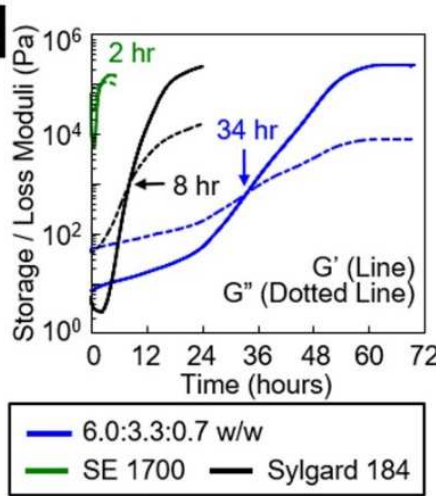

g

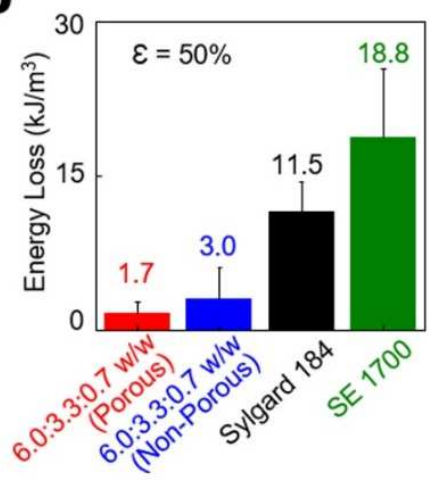

b

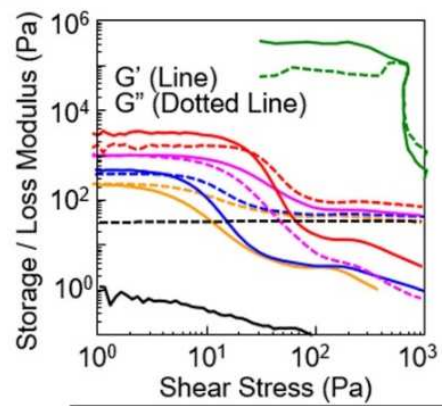

Shear Stress $(\mathrm{Pa})$
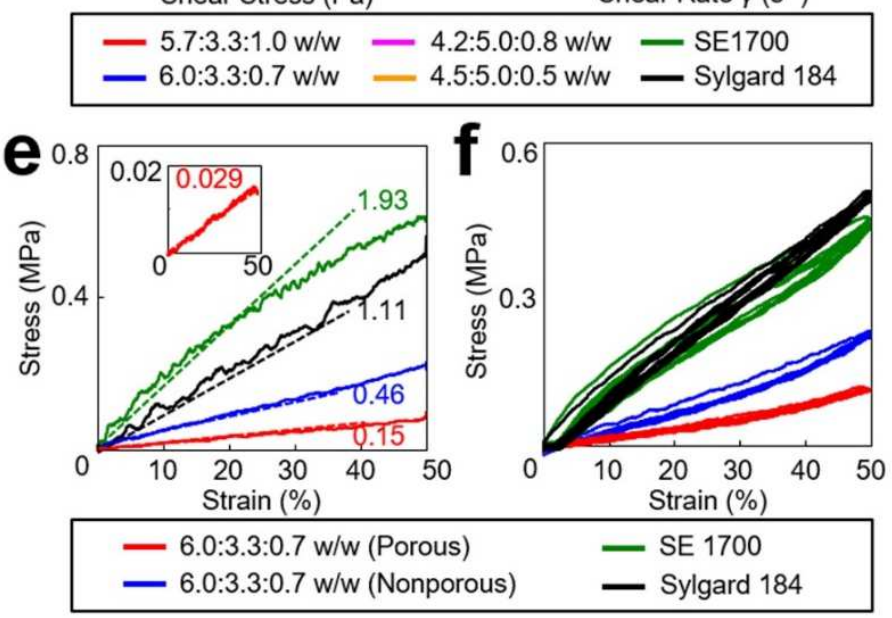

h
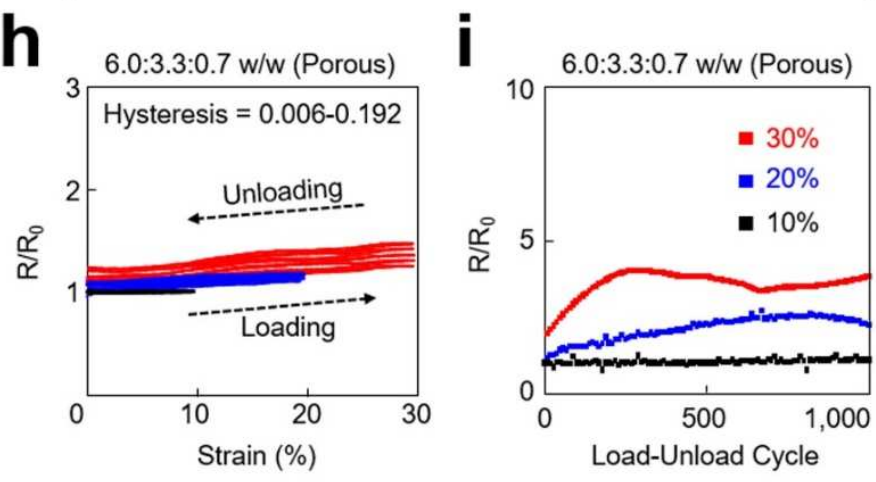

Figure 2. Material structure-property relationships. (a) Snapshot image of the MD simulation for the interfacial interaction energy of a $\mathrm{SiO}_{2}$-PS particle (left panel) and a $\mathrm{SiO}_{2}-\mathrm{OH}$ particle (right panel) with the surrounding resins. (b) Storage and loss modulus of the inks with respect to shear stress. (c) Viscosity of the inks with respect to shear rate. (d) Change in the storage and loss modulus of the inks over time. (e) Stress-strain curves of the inks. Inset graph shows the corresponding results obtained from the ink with 6.0:3.3:0.7 ratio configured into an open mesh layout. (f) Mechanical hysteresis of the inks for 5 loadingunloading cycles at a strain of 50\%. (g) Energy loss of the inks. (h) Electrical hysteresis of the inks with respect to the strain from $10 \%$ to $50 \%$. (i) Change in the resistance of the inks throughout 1,000 times of loading-unloading cycles with the strains ranging from $10 \%$ to $30 \%$. 

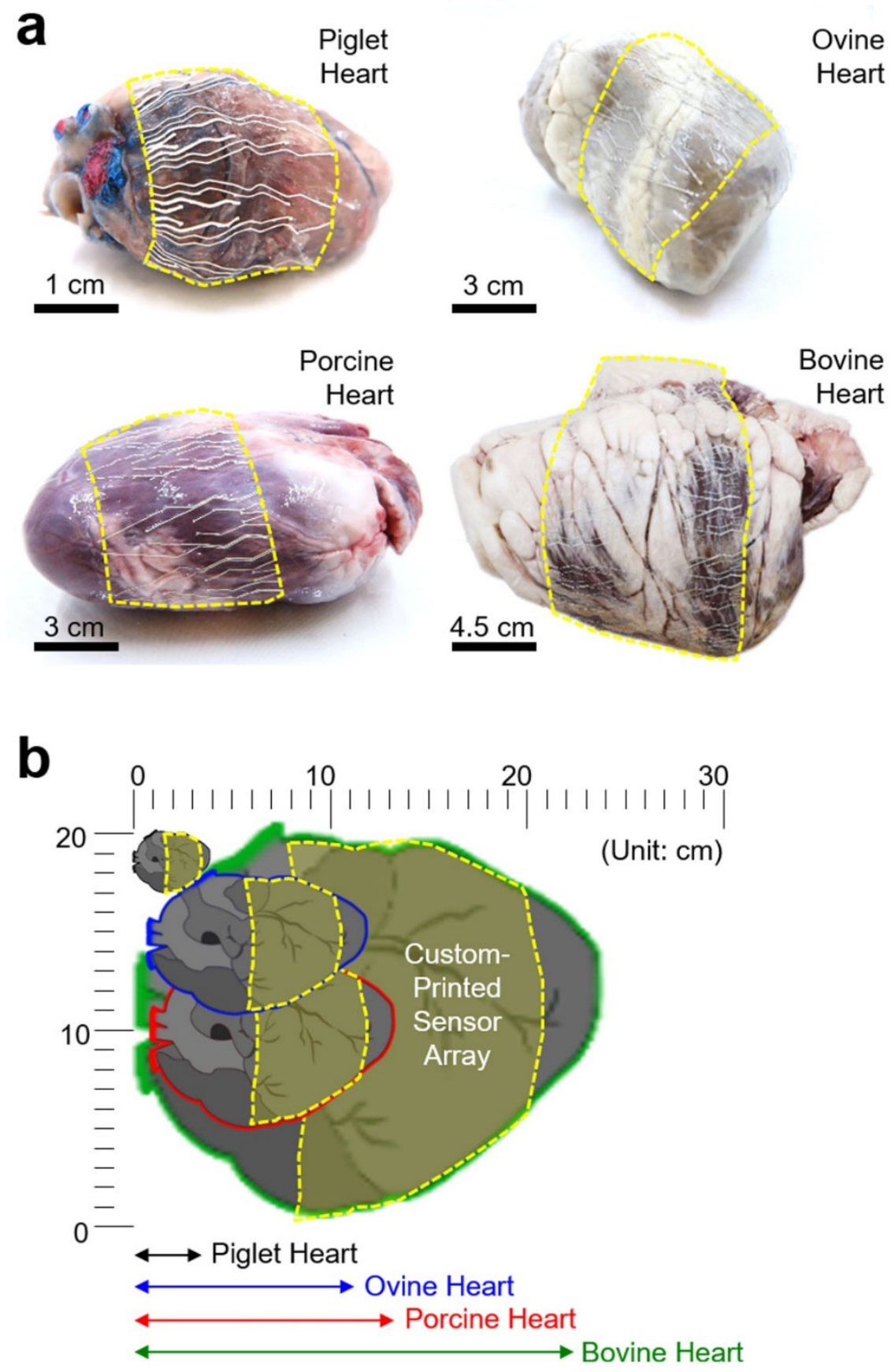

Figure 3. Rapid custom prototyping. (a) Photographs of the custom-printed sensor arrays, each of which was customized to fit with the enucleated piglet, ovine, porcine, and bovine hearts from upper left to bottom right clockwise. (b) Schematic illustration of the cross-sectional view of the hearts. 


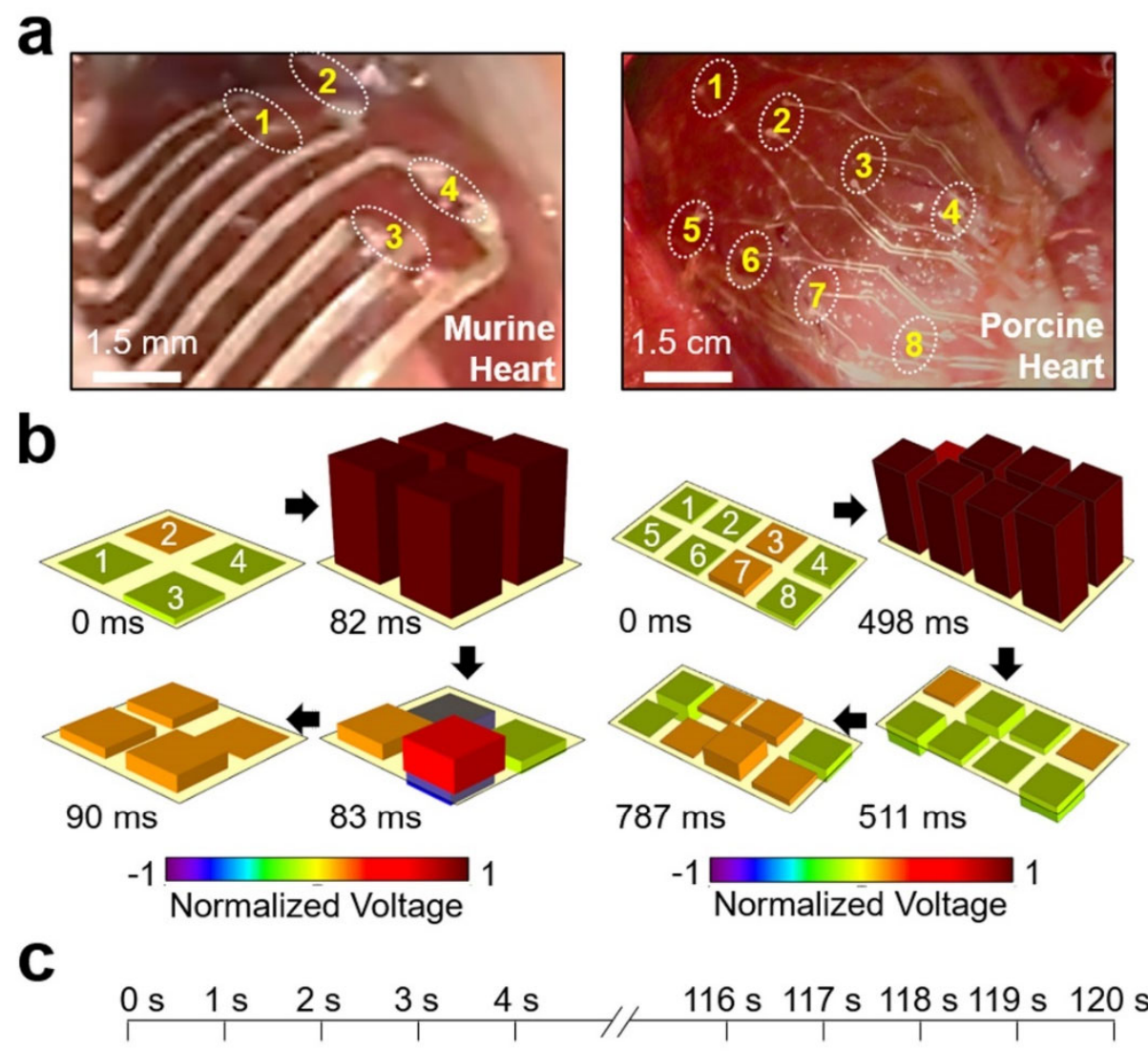

Custom-Printed Cardiac Sensor Patch In Mouse

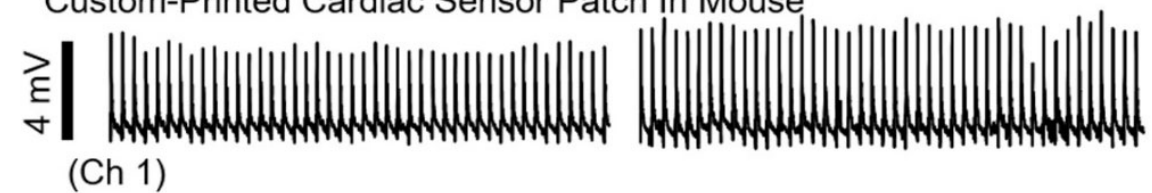

3-Lead Electrode Setting (Control)

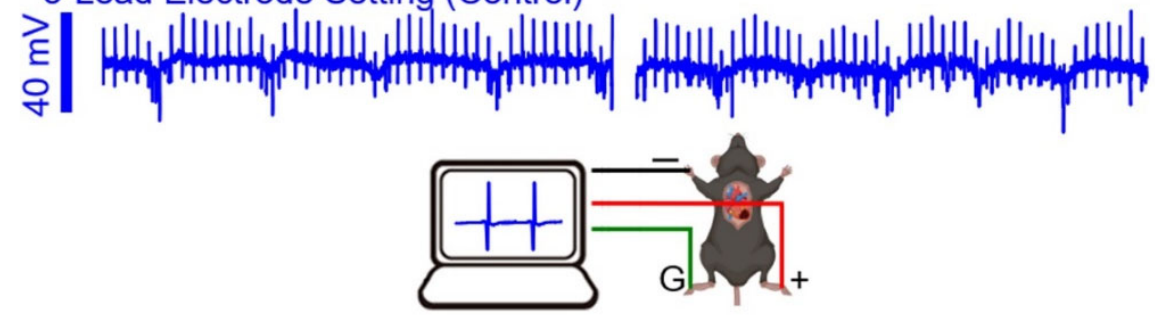

Figure 4. Spatiotemporal recording of epicardial ECG signals in vivo. (a) Photographs of the customprinted sensor arrays placed on the epicardial surface of a murine (left panel) and porcine heart (right panel). (b) Post-processed 3D data of the spatiotemporally recorded murine (left panel) and porcine (right panel) ECG signals. (c) Simultaneously measured ECG signals using the custom-printed sensor array (top panel) and a control 3-lead electrode set (bottom panel) on a murine heart. 


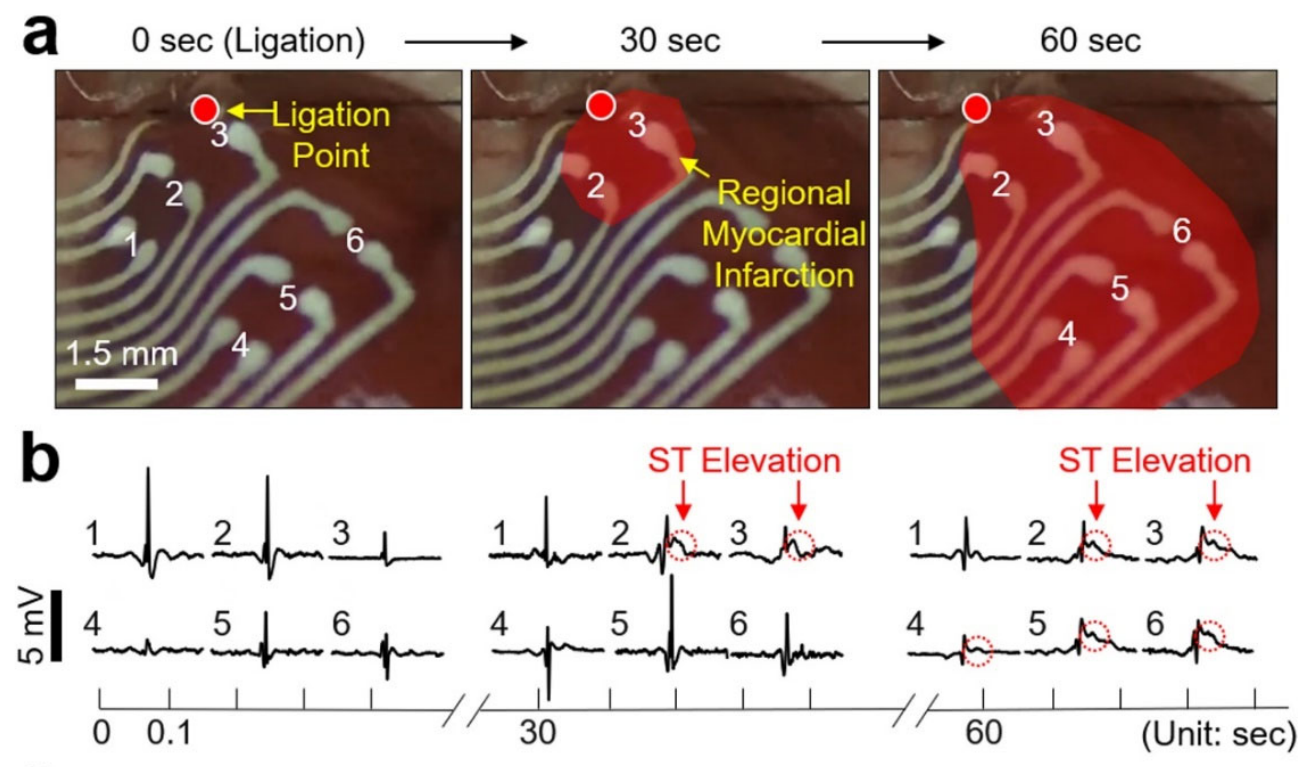

C

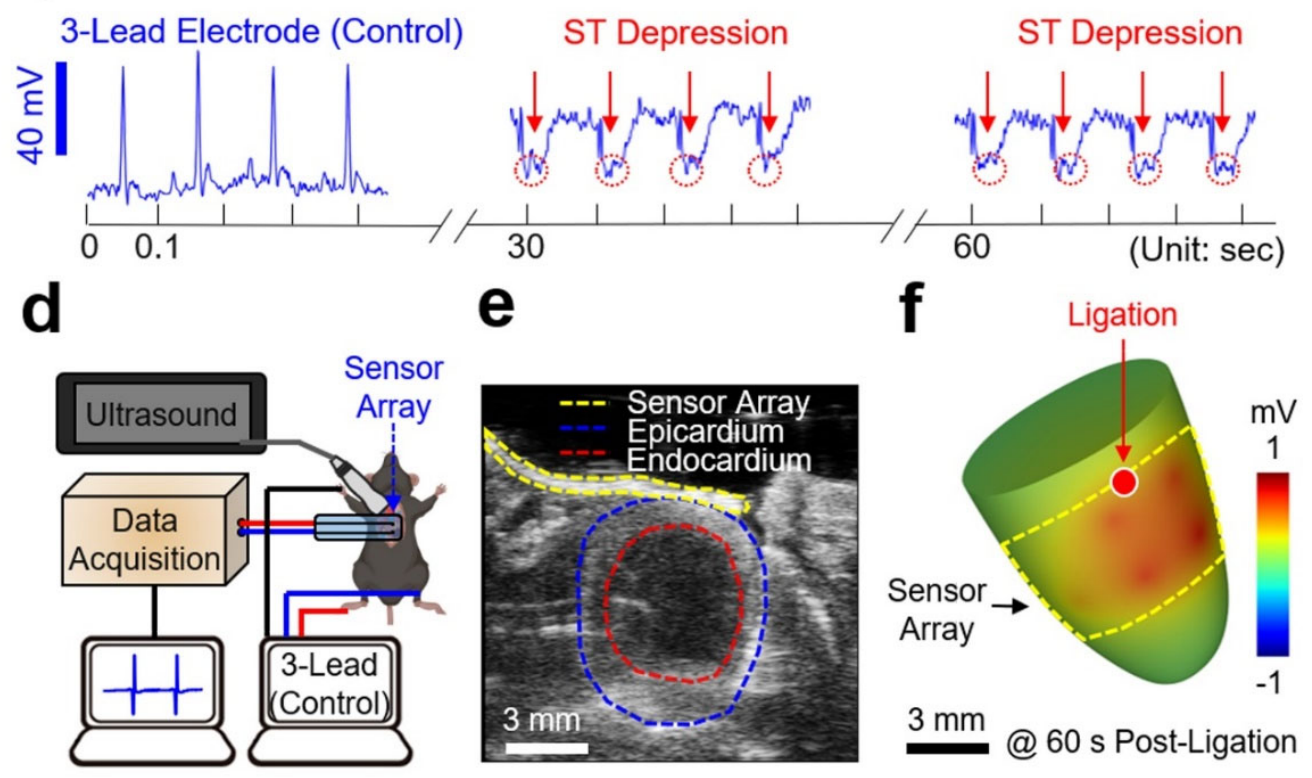

Figure 5. Intraoperative epicardial mapping in a murine myocardial infarction model. (a) Enlarged images of the custom-printed sensor array covering the entire ventricular epicardium. The red highlighted area indicates the regional myocardial infarction propagating toward the apex of the murine heart over time. (b) Measured epicardial ECG signals using the custom-printed sensor array. (c) Simultaneously measured ECG signals using a control 3-lead electrode set. (d) Schematic illustration of the experiment setup for simultaneous epicardial ECG recording and ultrasound imaging. (e) Representative short-axis ultrasound image displaying the custom-printed sensor array (yellow circle) along with the epicardium (blue circle) and endocardium (red circle) of the heart. (f) Post-processed 3D image reconstructed from the spatiotemporally recorded epicardial ECG and ultrasound signals after 60 seconds post-ligation. 

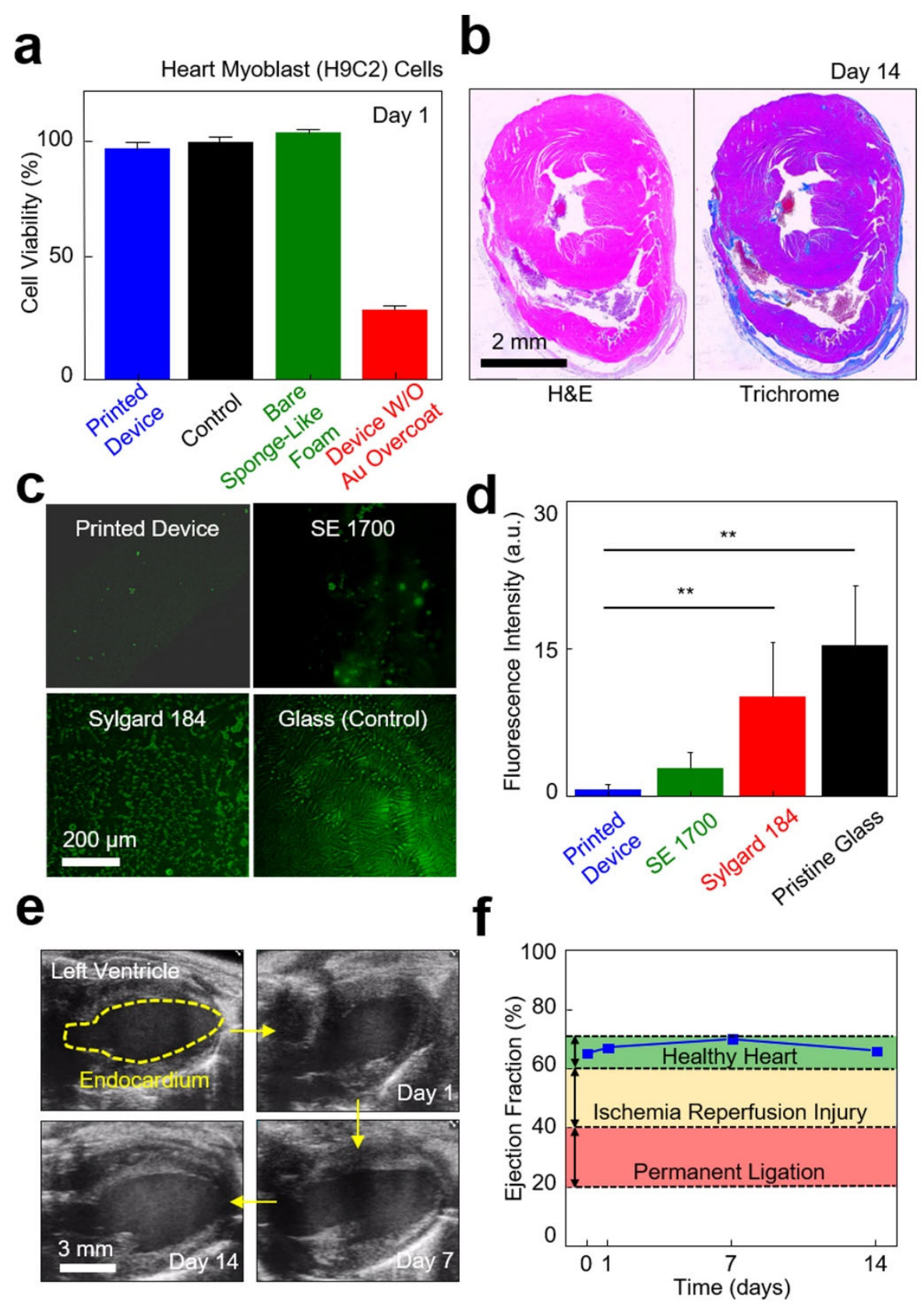

Figure 6. Evaluation of biocompatibility, anti-biofouling, and effect on cardiac function. (a) Results of cell compatibility assay for the custom-printed sensor array with heart myoblast (H9C2) cells in a 24well plate, as measured using a colorimetric assay kit. (b) Representative histological cross-sectional views of the murine cardiac tissues that were stained with both hematoxylin-eosin (H\&E; left panel) and Masson's trichrome (MTC; right panel) on day 14 post-implant of the custom-printed sensor array. (c) Z-stack fluorescence images of a BSA-FITC $(6 \mathrm{mg} / \mathrm{ml})$ coated on the printed device as compared to control groups made of the SE 1700 ink and the Sylgard 184 ink and prepared on a pristine glass, from upper left to bottom right clockwise. (d) Results of one-way ANOVA tests with Bonferroni correction ( $\mathrm{n}=5$ per group). Fluorescence intensity is shown as average \pm standard deviation $\left({ }^{* *} \mathrm{p}<0.001\right)$. (e) Representative ultrasound images of the left ventricle of a murine heart on $0,1,7$, and 14 days implantation of the custom-printed sensor array. (f) Results of the ejection fraction of the murine heart post-implant. 
Supplementary Figures 

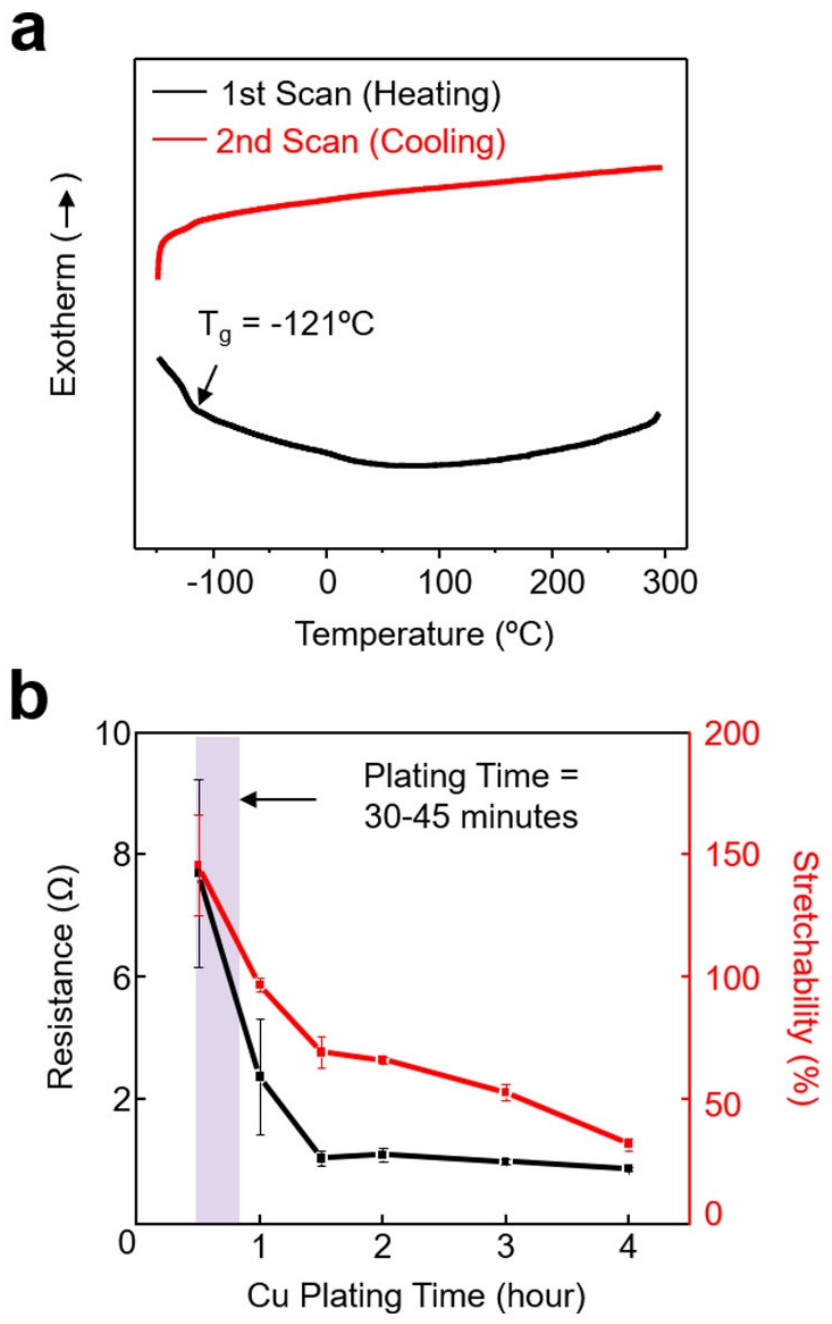

Figure S1. (a) Results of differential scanning calorimetry (DSC) for the sponge-like foam. (b) Change in the resistance and stretchability of the custom-printed sensor array with respect to the plating time of $\mathrm{Cu}$. 


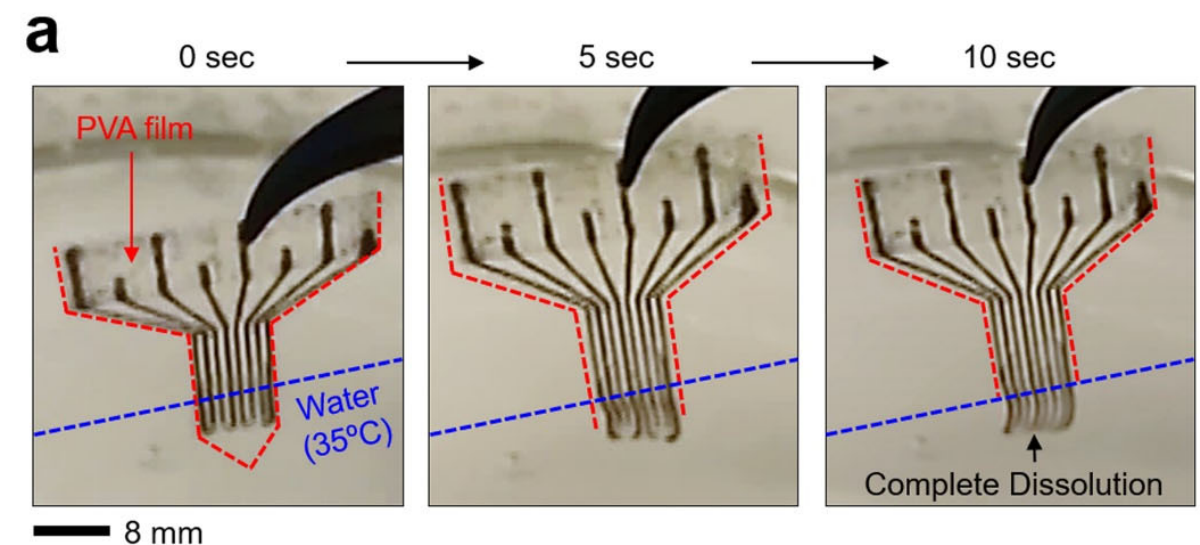

b

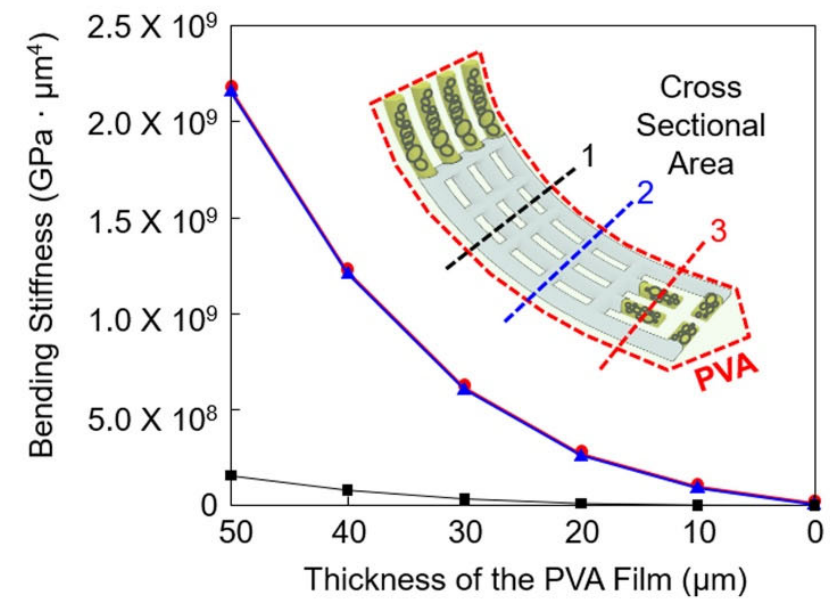

Figure S2. (a) Photographs of the custom-printed sensor array immersed in a warm water bath $\left(35^{\circ} \mathrm{C}\right)$ for 10 seconds. (b) Results of the bending stiffness of the custom-printed sensor array with respect to the thickness of the water-soluble medical tape. 


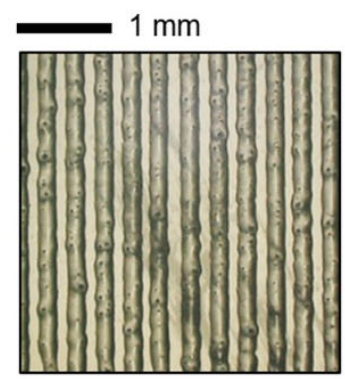

Linewidth $=200 \mu \mathrm{m}$

Spacing $=300 \mu \mathrm{m}$

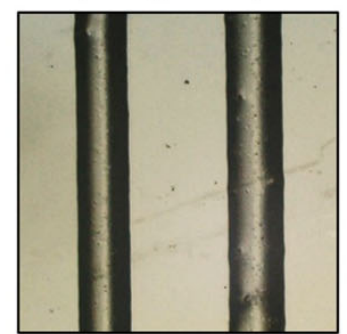

Linewidth $=500 \mu \mathrm{m}$

Spacing $=1,600 \mu \mathrm{m}$

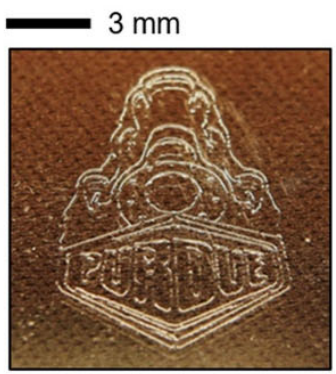

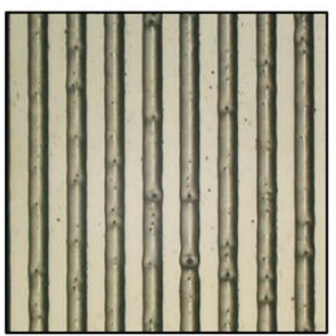

Linewidth $=200 \mu \mathrm{m}$

Spacing $=400 \mu \mathrm{m}$

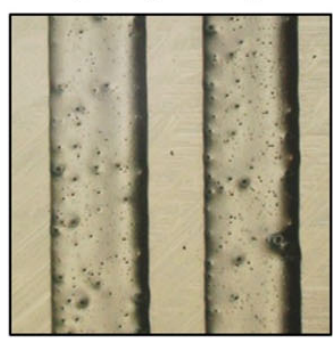

Linewidth $=800 \mu \mathrm{m}$

Spacing $=1,600 \mu \mathrm{m}$

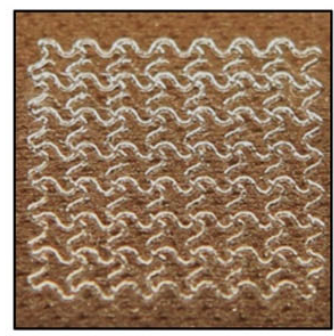

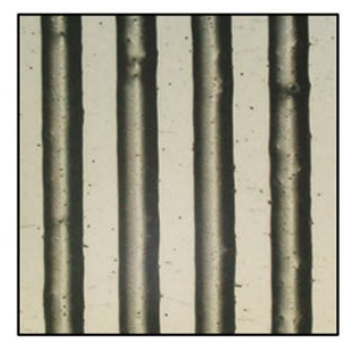

Linewidth $=300 \mu \mathrm{m}$ Spacing $=800 \mu \mathrm{m}$

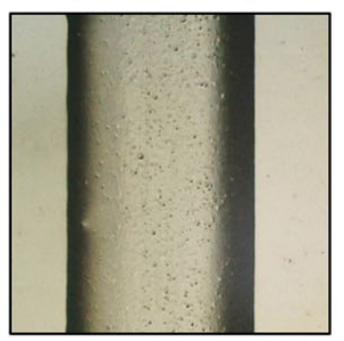

Linewidth $=1,800 \mu \mathrm{m}$ Spacing $=3,200 \mu \mathrm{m}$

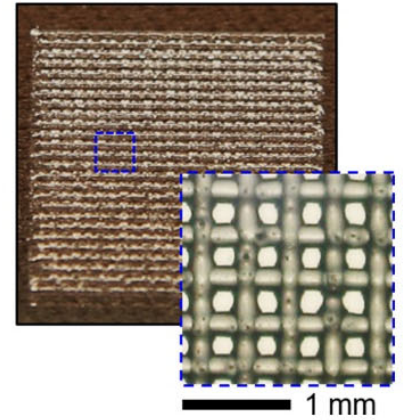

Figure S3. Photographs of various as-printed structures using the formulated ink with the weight ratio of 6.0:3.3:0.7. 


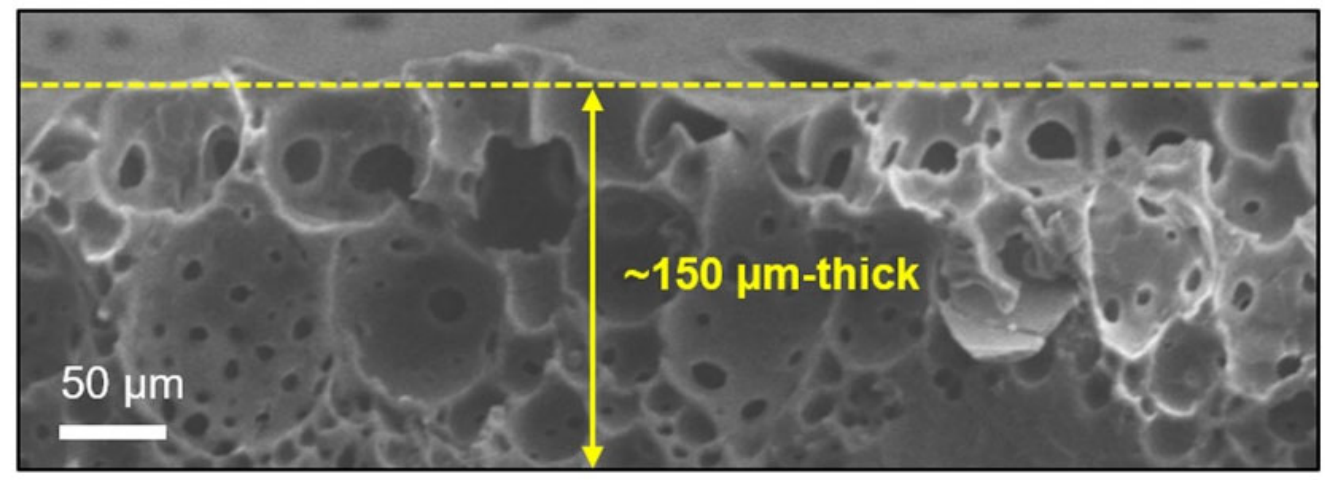

Figure S4. Cross-sectional side view of a SEM image for the sponge-like foam.

Formulated Ink (6.0:3.3:0.7 w/w)

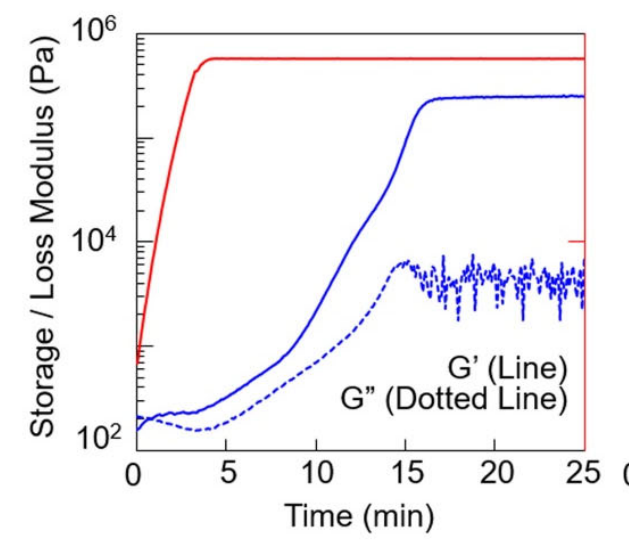

Formulated Ink (6.0:3.3:0.7 w/w) with Directly Mixed Ag Flakes

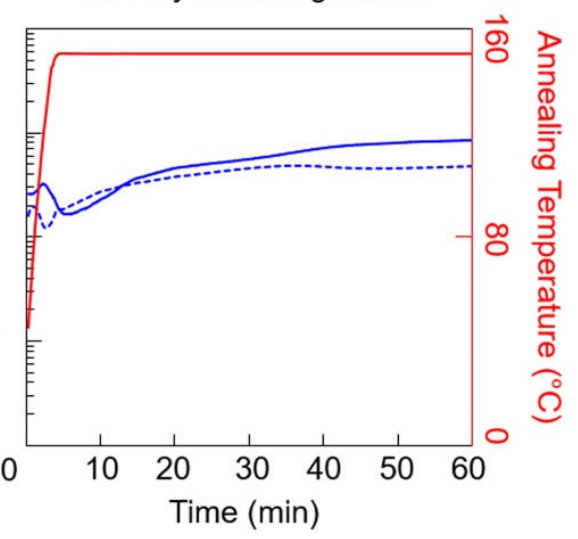

Figure S5. Change in the storage and loss modulus of the formulated ink with the weight ratio of 6.0:3.3:0.7 (left panel) and the ink with directly mixed Ag flakes (right panel) over time. 


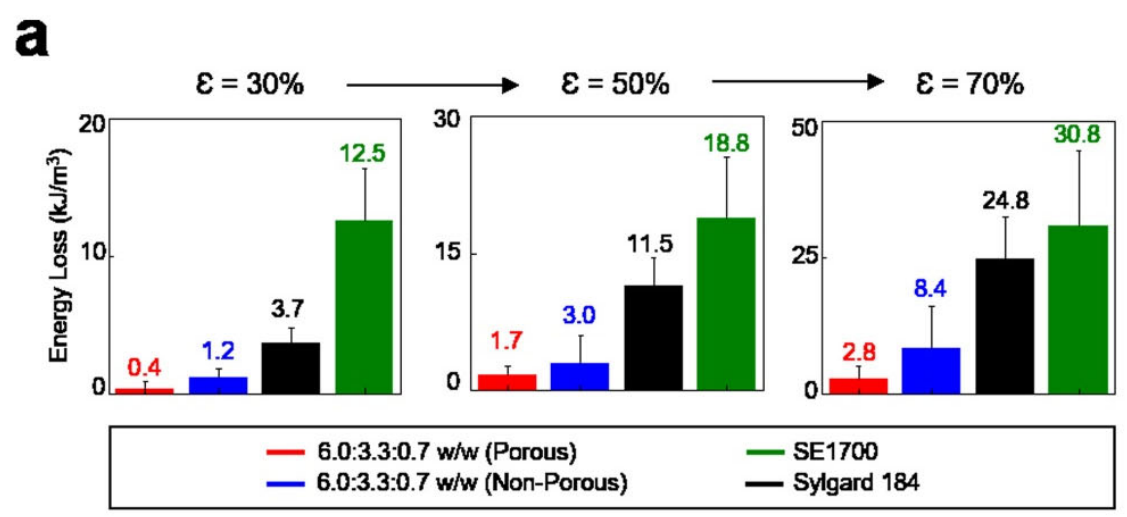

b

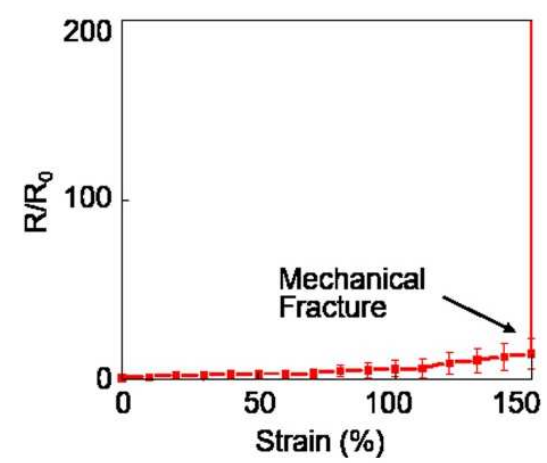

Figure S6. (a) Change in the energy loss of the custom-printed sensor array with respect to a strain ranging from $30 \%$ to $70 \%$. (b) Change in the resistance of the custom-printed sensor array with respect to the strain up to $150 \%$. 

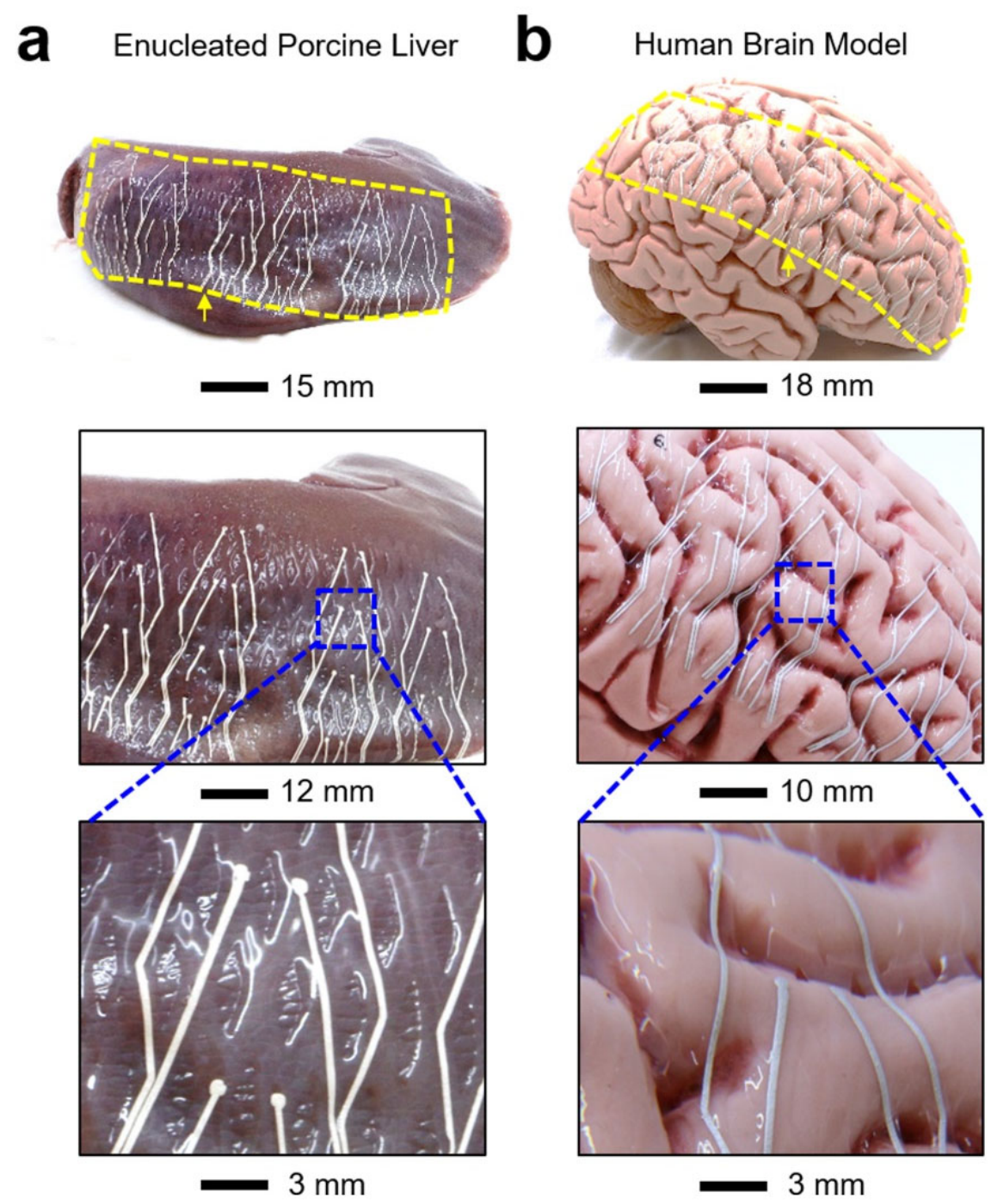

Figure S7. Photographs of the custom-printed sensor array placed on the surface of (a) the enucleated porcine liver and (b) human brain model. 


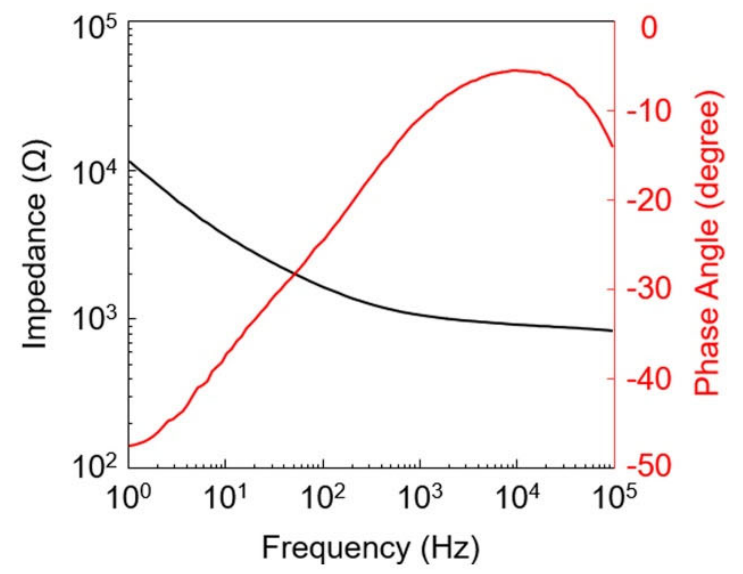

Figure S8. Electrochemical impedance of the custom-printed sensor array in a phosphate-buffered saline with respect to frequency.
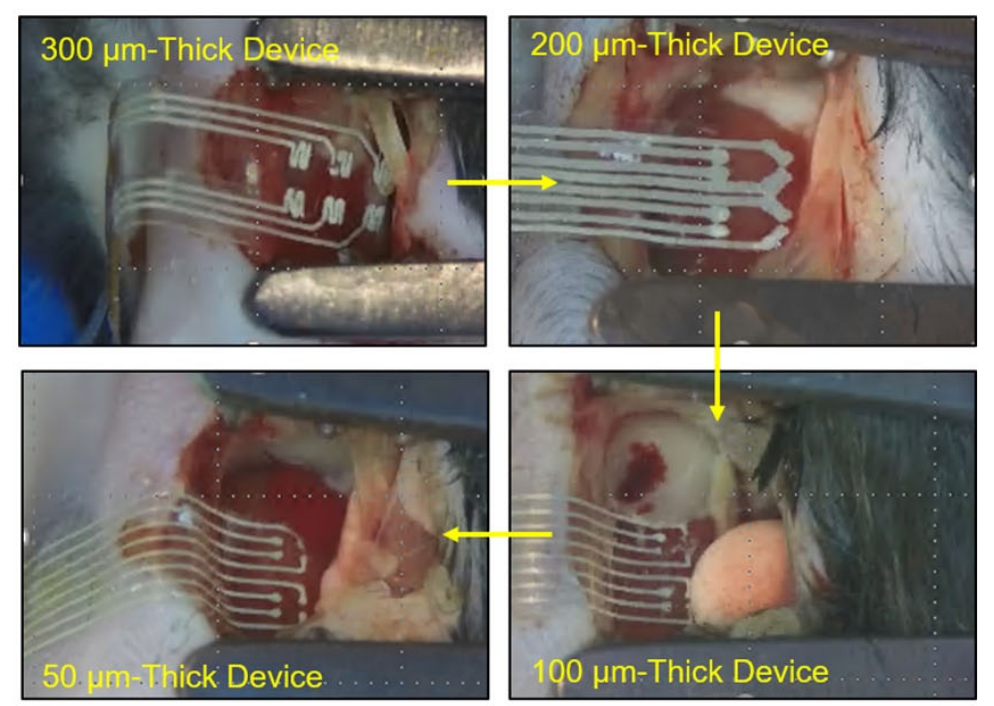

$5 \mathrm{~mm}$

Figure S9. Photographs of the custom-printed sensor arrays with different thicknesses ranging from $50 \mu \mathrm{m}$ to $300 \mu \mathrm{m}$ placed on the epicardial surface of a murine heart. 
Murine Epicardial ECG
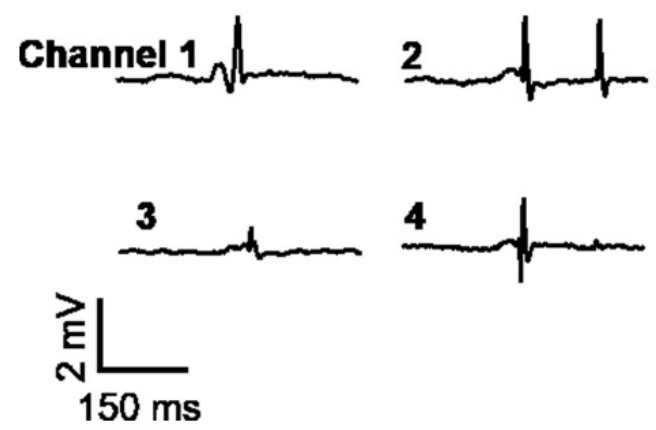

Porcine Epicardial ECG
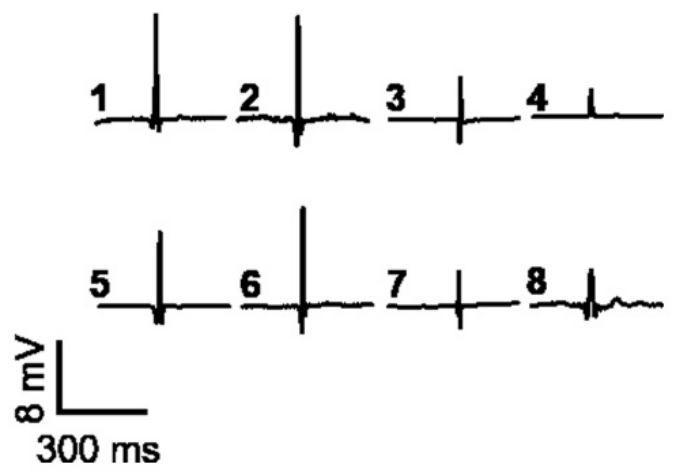

Figure S10. Murine (left panel) and porcine (right panel) ECG signals measured using the custom-printed devices.

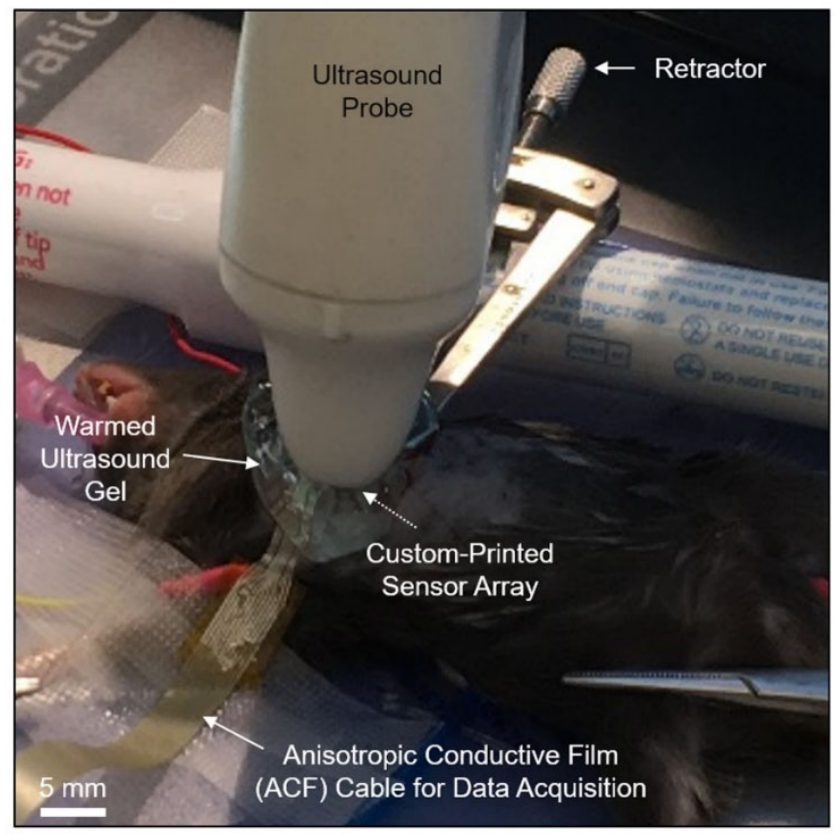

Figure S11. Photograph of the measurement setup for simultaneous epicardial ECG recording and ultrasound imaging. 

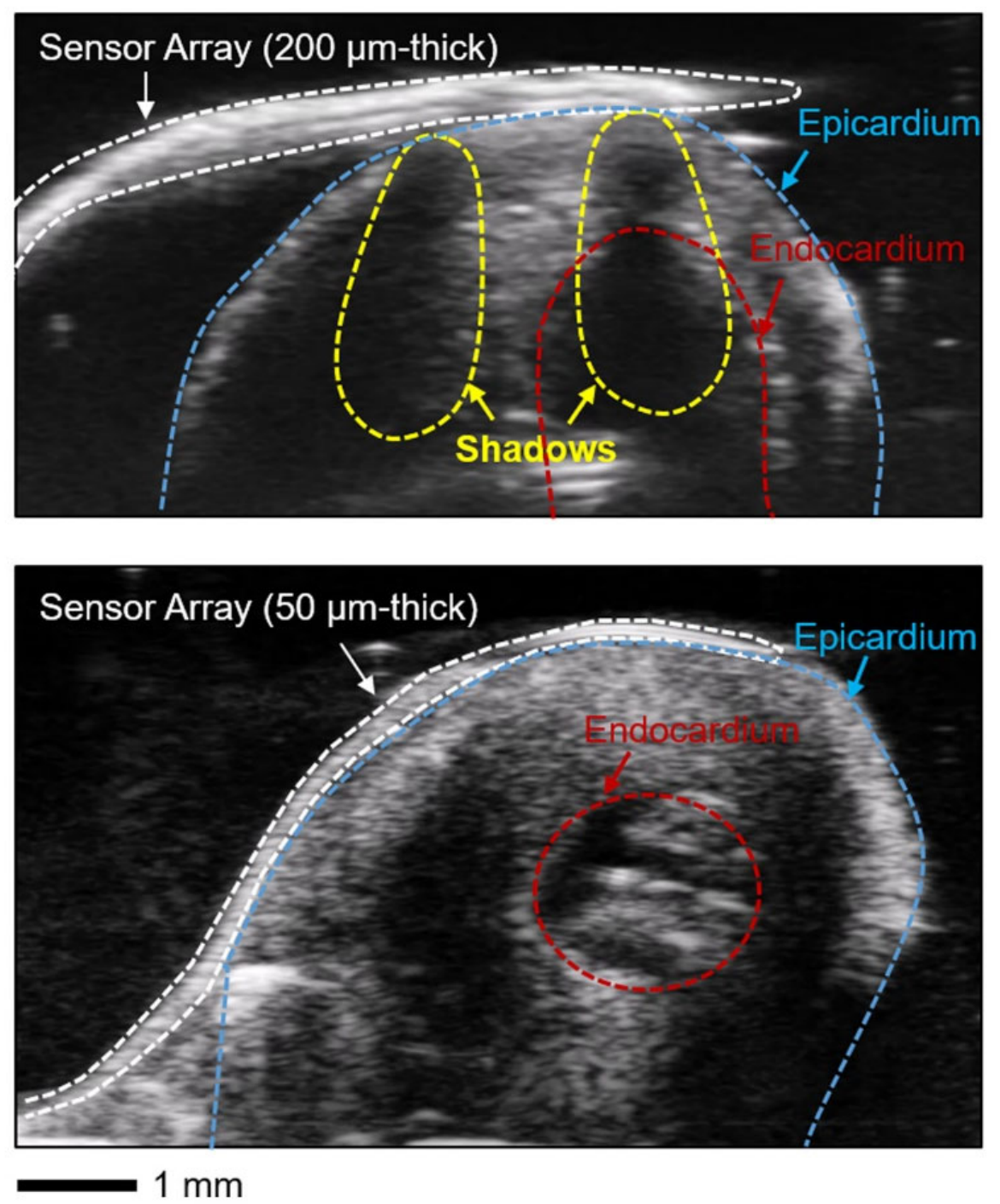

Figure S12. Representative ultrasound images of the relatively thick (200 $\mu$ m-thick; top panel) and thin (50 $\mu \mathrm{m}$-thick; bottom panel) devices placed on the epicardial surface of a fixed murine heart. 

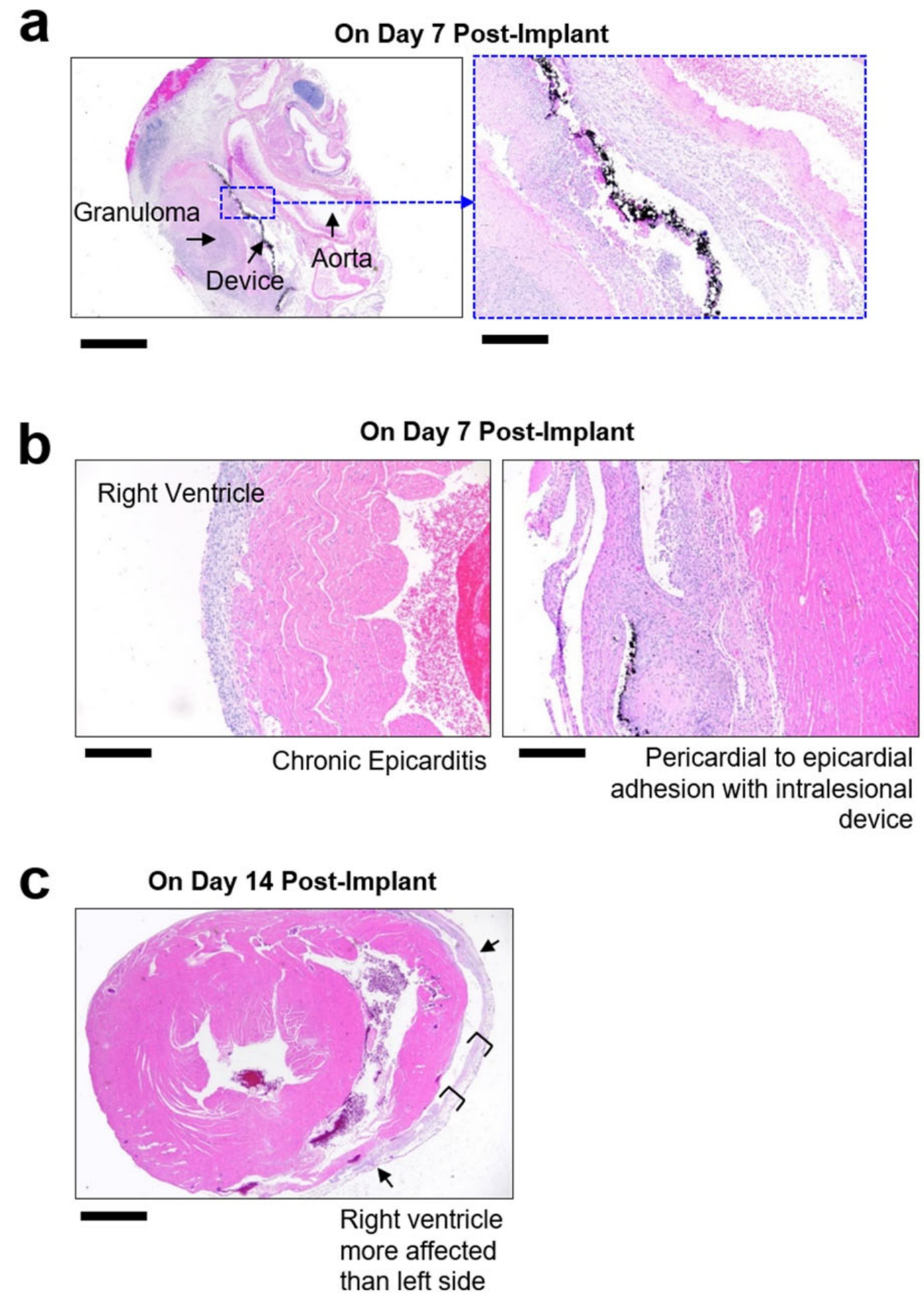

Figure S13. Representative histological cross-sectional views of the murine cardiac tissues that were stained with both hematoxylin-eosin (H\&E) and Masson's trichrome (MTC) on day 7 and day 14 postimplant of the custom-printed sensor array. 


\section{Supplementary Table}


Table S1. Comparison of the MD simulation results for the interfacial interaction energy of a $\mathrm{SiO}_{2}-\mathrm{PS}$ particle and a $\mathrm{SiO}_{2}-\mathrm{OH}$ particle with the surrounding resins.

\begin{tabular}{|c|c|c|c|c|c|c|}
\hline \multirow[b]{2}{*}{ Silica Type } & \multirow[b]{2}{*}{ Surface Property } & \multicolumn{3}{|c|}{$\begin{array}{l}\text { Interaction Energy } \\
\text { (kcal/mol) }\end{array}$} & \multirow{2}{*}{$\begin{array}{c}\text { Interaction } \\
\text { Energy Per } \\
\text { Area } \\
\left(\mathrm{kcal} / \mathrm{mol} \mathrm{A} \mathrm{A}^{2}\right)\end{array}$} & \multirow{2}{*}{$\begin{array}{c}\text { Interaction } \\
\text { Energy Per Silica } \\
\text { Weight } \\
\text { (kcal/mol g-silica) }\end{array}$} \\
\hline & & $\begin{array}{c}\text { Total } \\
\text { Energy }\end{array}$ & $\begin{array}{l}\text { Van der } \\
\text { Waals } \\
\text { Energy }\end{array}$ & $\begin{array}{c}\text { Electrostatic } \\
\text { Energy }\end{array}$ & & \\
\hline $\mathrm{SiO}_{2}-\mathrm{PS}$ & uperhydrophobic & -119.1 & -118.8 & -0.3 & -0.1610 & $-1.852 \times 10^{21}$ \\
\hline $\mathrm{SiO}_{2}-\mathrm{OH}$ & Hydrophilic & -160.4 & -149.3 & -8.0 & -0.2144 & $-6.969 \times 10^{21}$ \\
\hline
\end{tabular}




\section{Figures}

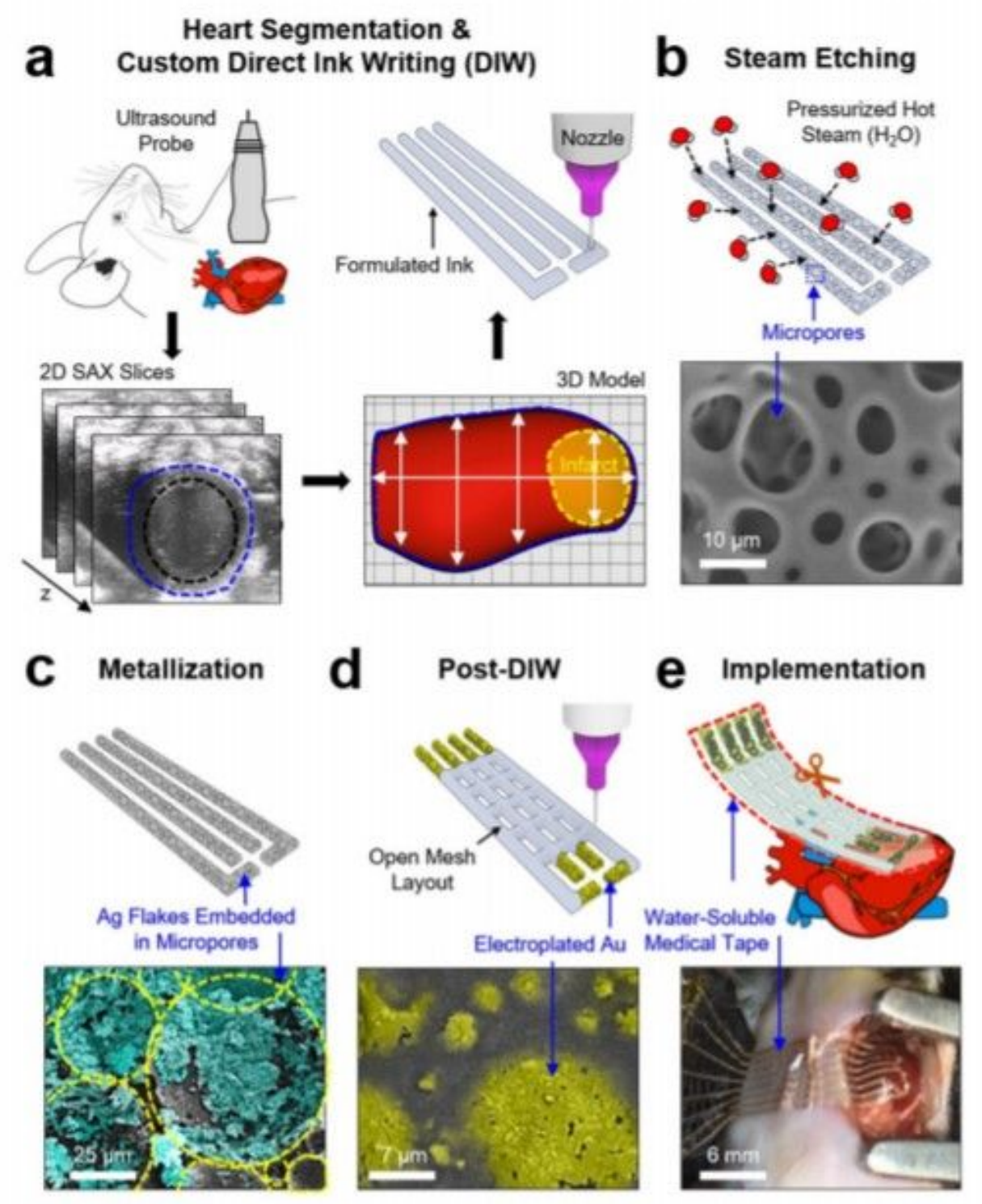

Figure 1

Custom design and prototyping of poroelastic biosensor array. Schematic illustrations and optical images for the key process steps: (a) 3D imaging and custom direct ink writing (DIW), (b) steam etching, (c) metallization, (d) post-DIW, and (e) implementation on the epicardial surface of a murine heart. 


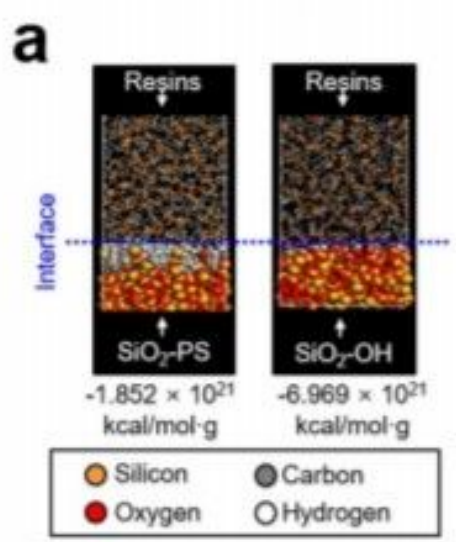

b

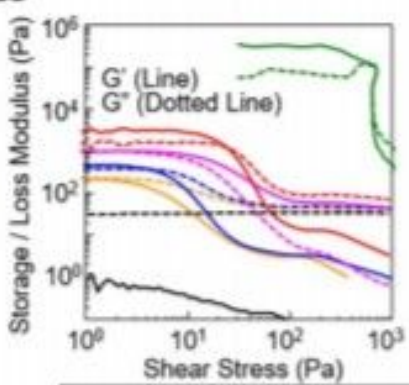

C

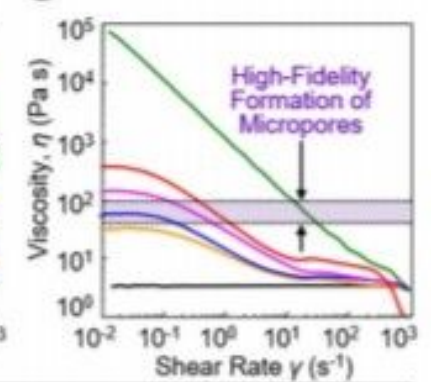

- 5.7:3.3:1.0 w/w $-4 \cdot 2 \cdot 5 \cdot 0 \cdot 0.8 \mathrm{wiw}-\mathrm{SE} 1700$ - 6.0:3.3:0.7 wiw - 4.5:5.0.0.5 w/w - Sylgard 184
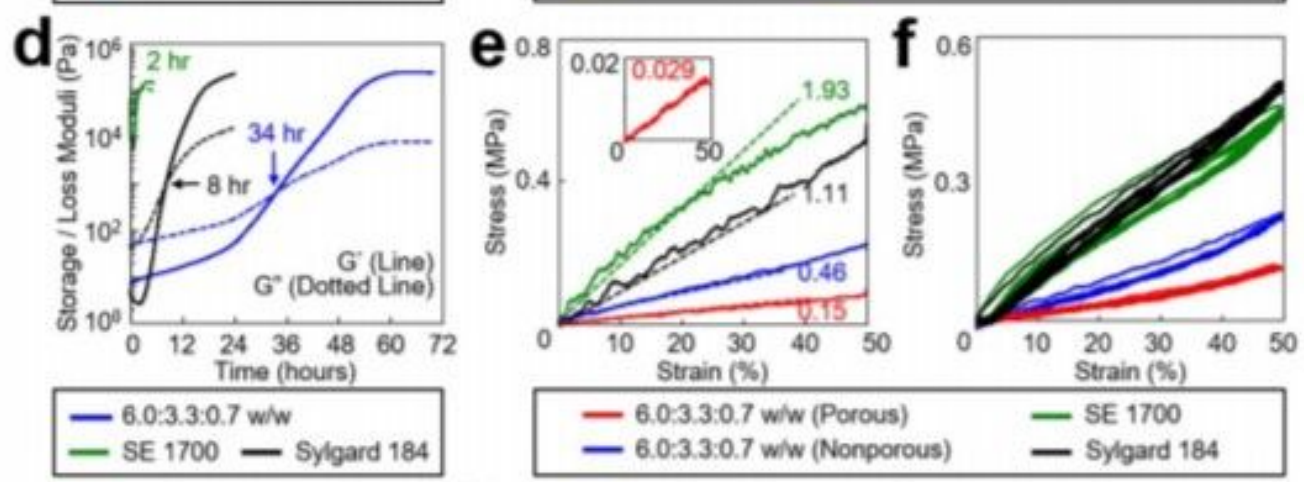

g

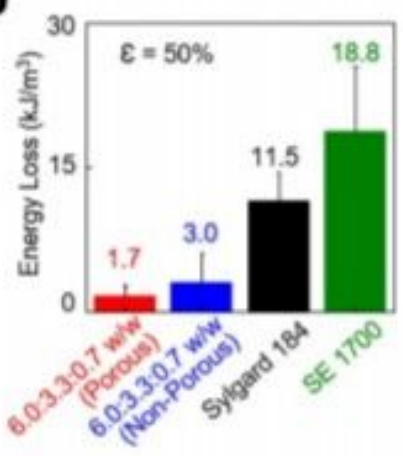

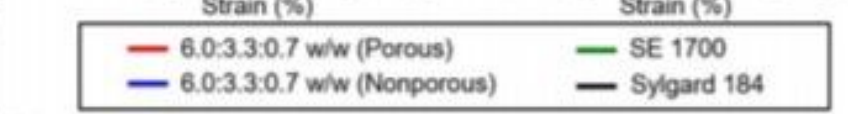

h

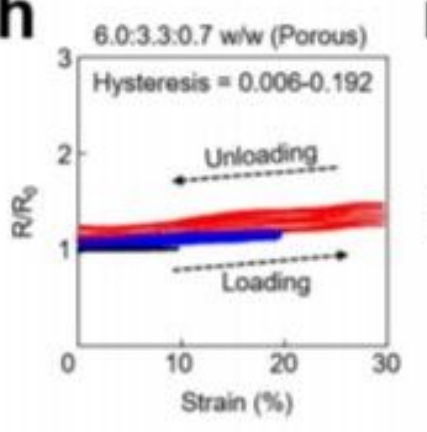

\section{Figure 2}

Material structure-property relationships. (a) Snapshot image of the MD simulation for the interfacial interaction energy of a SiO2-PS particle (left panel) and a SiO2-OH particle (right panel) with the surrounding resins. (b) Storage and loss modulus of the inks with respect to shear stress. (c) Viscosity of the inks with respect to shear rate. (d) Change in the storage and loss modulus of the inks over time. (e) Stress-strain curves of the inks. Inset graph shows the corresponding results obtained from the ink with 6.0:3.3:0.7 ratio configured into an open mesh layout. (f) Mechanical hysteresis of the inks for 5 loadingunloading cycles at a strain of 50\%. (g) Energy loss of the inks. (h) Electrical hysteresis of the inks with respect to the strain from $10 \%$ to $50 \%$. (i) Change in the resistance of the inks throughout 1,000 times of loading-unloading cycles with the strains ranging from $10 \%$ to $30 \%$. 

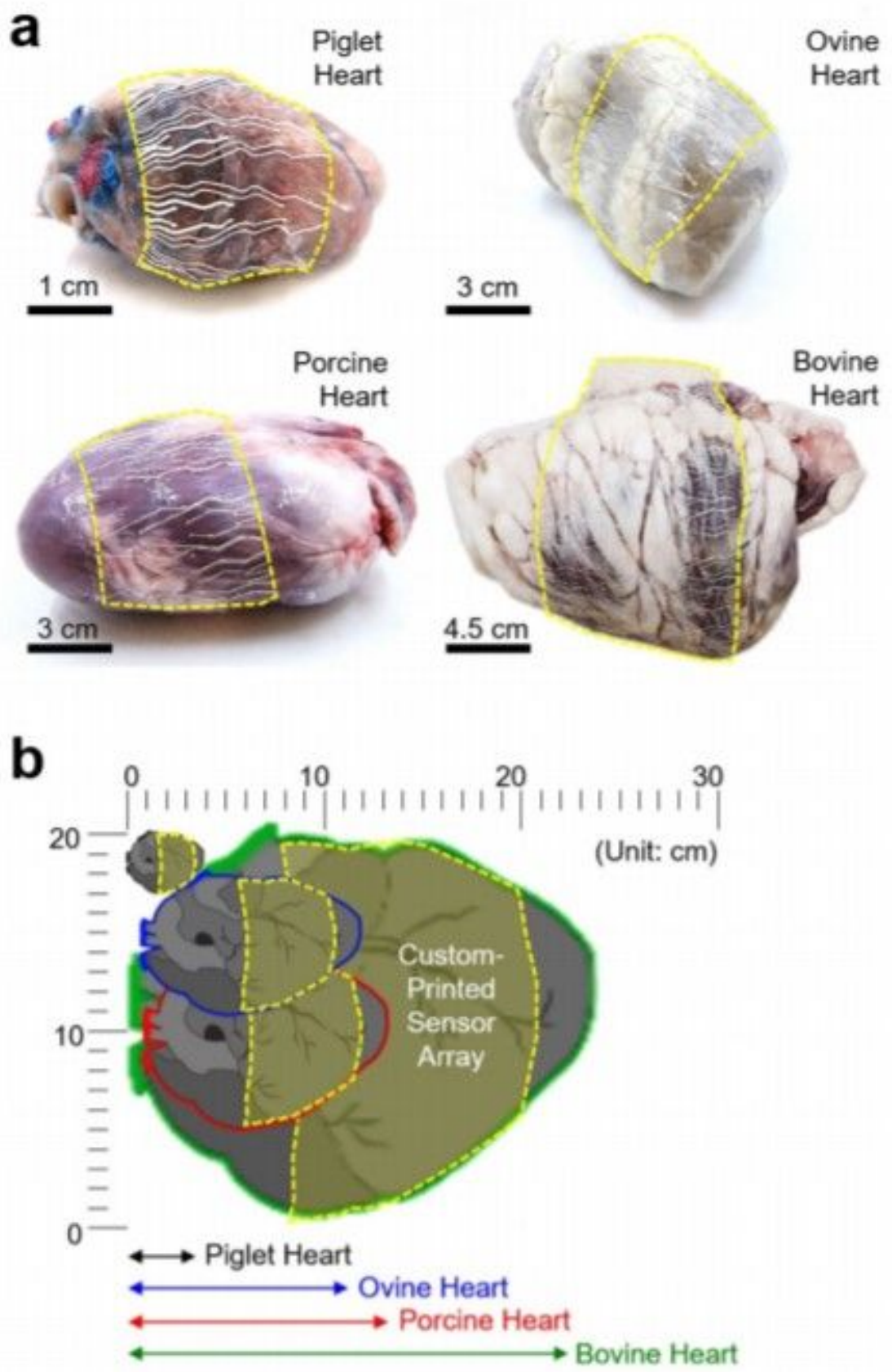

\section{Figure 3}

Rapid custom prototyping. (a) Photographs of the custom-printed sensor arrays, each of which was customized to fit with the enucleated piglet, ovine, porcine, and bovine hearts from upper left to bottom right clockwise. (b) Schematic illustration of the cross-sectional view of the hearts. 

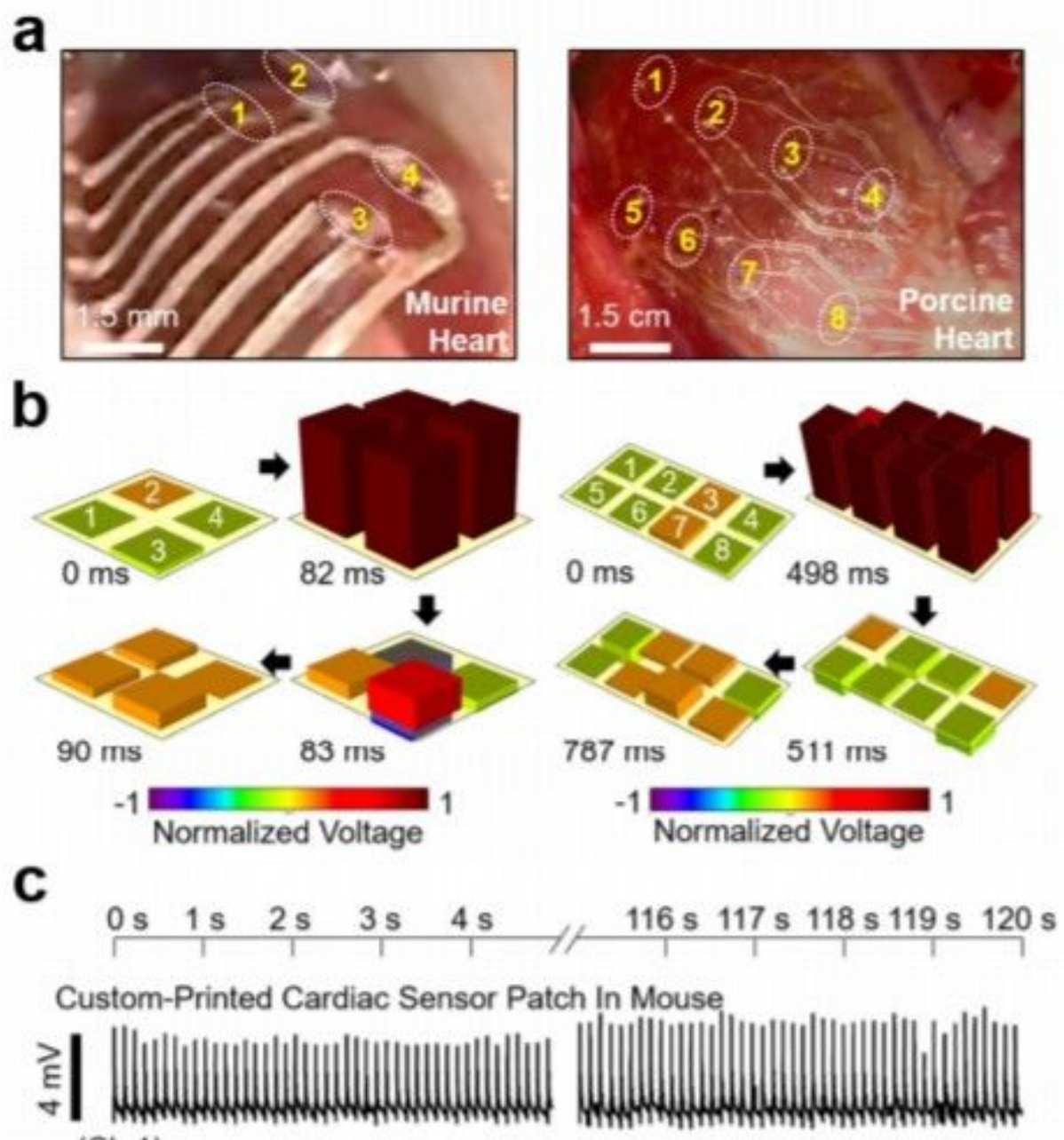

(Ch 1)

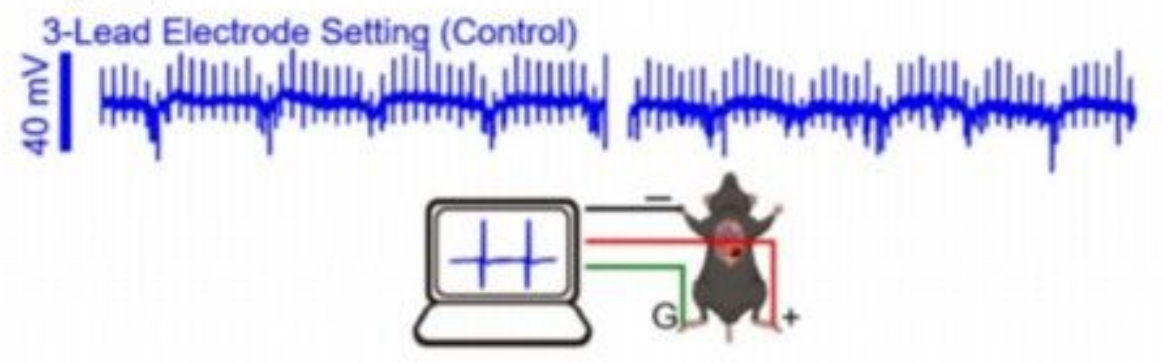

Figure 4

Spatiotemporal recording of epicardial ECG signals in vivo. (a) Photographs of the customprinted sensor arrays placed on the epicardial surface of a murine (left panel) and porcine heart (right panel). (b) Postprocessed 3D data of the spatiotemporally recorded murine (left panel) and porcine (right panel) ECG signals. (c) Simultaneously measured ECG signals using the custom-printed sensor array (top panel) and a control 3-lead electrode set (bottom panel) on a murine heart. 


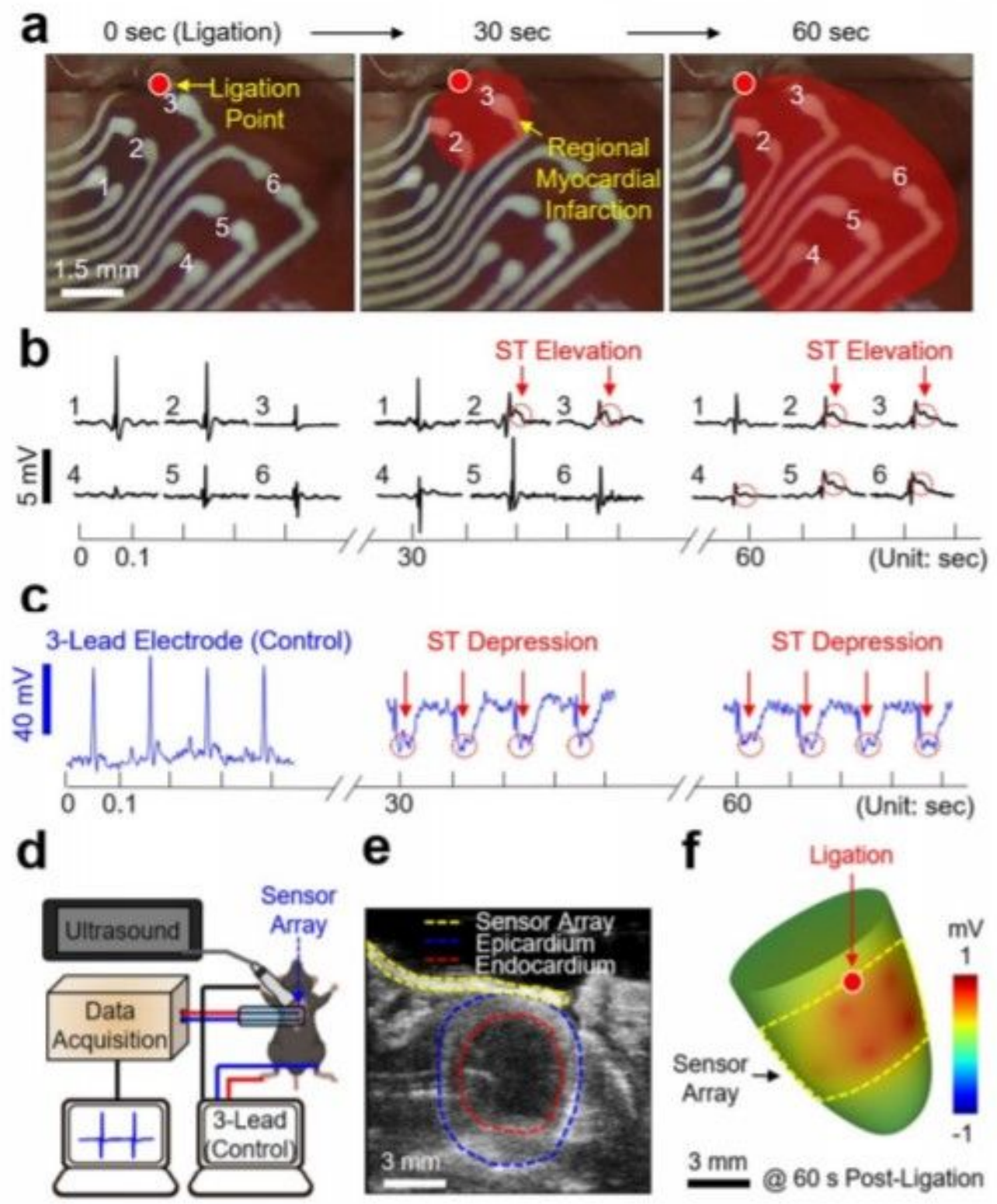

\section{Figure 5}

Intraoperative epicardial mapping in a murine myocardial infarction model. (a) Enlarged images of the custom-printed sensor array covering the entire ventricular epicardium. The red highlighted area indicates the regional myocardial infarction propagating toward the apex of the murine heart over time. (b) Measured epicardial ECG signals using the custom-printed sensor array. (c) Simultaneously measured ECG signals using a control 3-lead electrode set. (d) Schematic illustration of the experiment setup for simultaneous epicardial ECG recording and ultrasound imaging. (e) Representative short-axis ultrasound image displaying the custom-printed sensor array (yellow circle) along with the epicardium (blue circle) and endocardium (red circle) of the heart. (f) Post-processed 3D image reconstructed from the spatiotemporally recorded epicardial ECG and ultrasound signals after 60 seconds post-ligation. 


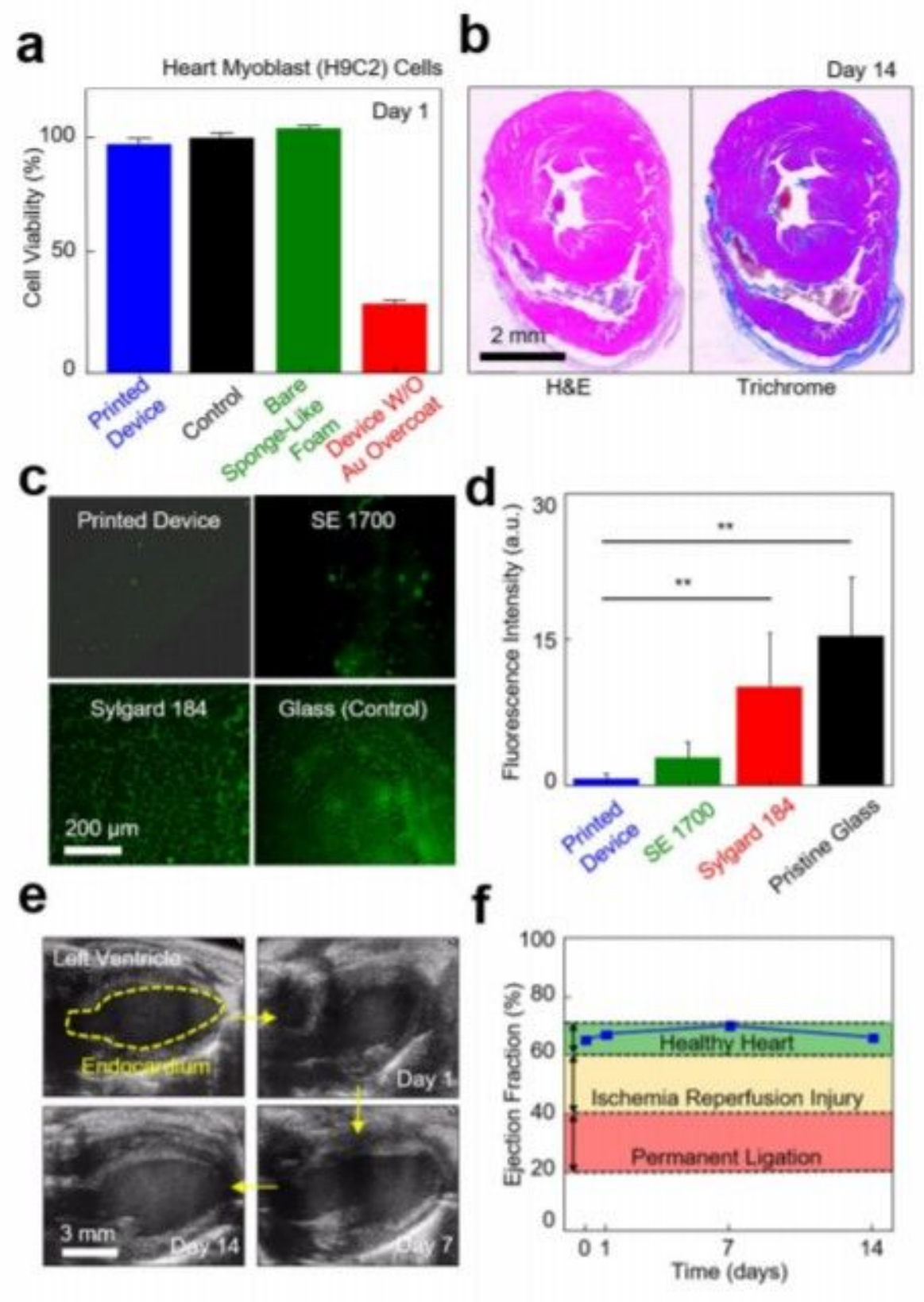

Figure 6

Evaluation of biocompatibility, anti-biofouling, and effect on cardiac function. (a) Results of cell compatibility assay for the custom-printed sensor array with heart myoblast ( $\mathrm{H} 9 \mathrm{C} 2)$ cells in a 24- well plate, as measured using a colorimetric assay kit. (b) Representative histological cross-sectional views of the murine cardiac tissues that were stained with both hematoxylin-eosin (H\&E; left panel) and Masson's trichrome (MTC; right panel) on day 14 post-implant of the custom-printed sensor array. (c) Z-stack fluorescence images of a BSA-FITC $(6 \mathrm{mg} / \mathrm{ml})$ coated on the printed device as compared to control groups made of the SE 1700 ink and the Sylgard 184 ink and prepared on a pristine glass, from upper left to bottom right clockwise. (d) Results of one-way ANOVA tests with Bonferroni correction ( $n=5$ per 
group). Fluorescence intensity is shown as average \pm standard deviation ( $* \star p<0.001)$. (e) Representative ultrasound images of the left ventricle of a murine heart on $0,1,7$, and 14 days implantation of the custom-printed sensor array. (f) Results of the ejection fraction of the murine heart post-implant.

\section{Supplementary Files}

This is a list of supplementary files associated with this preprint. Click to download.

- MovieS1.mp4

- Movies2.mp4

- MovieS3.mp4

- MovieS4.mp4

- MovisS5.mp4 